\title{
Singularly perturbed elliptic problems with superlinear or asymptotically linear nonlinearities
}

\author{
Louis Jeanjean* and Kazunaga Tanaka** \\ * Equipe de Mathématiques (UMR CNRS 6623) \\ Université de Franche-Comté \\ 16 Route de Gray, 25030 Besançon, France \\ jeanjean@math.univ-fcomte.fr \\ ** Department of Mathematics \\ School of Science and Engineering \\ Waseda University \\ 3-4-1 Ohkubo, Shinjuku-ku, Tokyo 169-8555, Japan \\ kazunaga@waseda.jp
}

\begin{abstract}
We consider a class of equations of the form

$$
-\varepsilon^{2} \Delta u+V(x) u=f(u), \quad u \in H^{1}\left(\mathbf{R}^{N}\right) .
$$

By variational methods, we show the existence of families of positive solutions concentrating around local minima of the potential $V(x)$, as $\varepsilon \rightarrow 0$. We do not require uniqueness of the ground state solutions of the associated autonomous problems nor the monotonicity of the function $\xi \mapsto \frac{f(\xi)}{\xi}$. We deal with asymptotically linear as well as superlinear nonlinearities.
\end{abstract}

\section{Introduction}

In this paper we study the existence of positive solutions of the equation

$$
-\varepsilon^{2} \Delta u+V(x) u=f(u), \quad u \in H^{1}\left(\mathbf{R}^{N}\right) .
$$

We assume $f \in C^{1}(\mathbf{R}, \mathbf{R})$ and that $V(x)$ is locally Hölder continuous and bounded below away from 0 , that is, there exists $V_{0}>0$ such that

$$
V(x) \geq V_{0}>0 \quad \text { for all } x \in \mathbf{R}^{N} .
$$


A basic motivation to study (0.1) stems from the nonlinear Schrödinger equation

$$
i \hbar \frac{\partial \Phi}{\partial t}=-\frac{\hbar^{2}}{2 m} \Delta \Phi+W(x) \Phi-g(|\Phi|) \Phi .
$$

We are interested in standing wave solutions, namely solutions of the form $\Phi(x, t)=$ $u(x) e^{-\frac{i E t}{\hbar}}$ and it is easily observed that a $\Phi(x, t)$ of this form satisfies $(0.3)$ if and only if $u(x)$ is a solution of (0.1) with $V(x)=W(x)-E, \varepsilon^{2}=\frac{\hbar^{2}}{2 m}$ and $f(u)=g(u) u$.

An interesting class of solutions of (0.1), sometimes called semi-classical states, are families of solutions $u_{\varepsilon}(x)$ which concentrate and develop a spike shape around one, or more, special points in $\mathbf{R}^{N}$, while vanishing elsewhere as $\varepsilon \rightarrow 0$.

The existence of single and multiple spike solutions was first studied by Floer and Weinstein $[\mathbf{F W}]$. In the one dimensional case and for $f(u)=u^{3}$ they construct a single spike solution concentrating around any given non-degenerate critical point of the potential $V(x)$. Oh $[\mathbf{O} 1, \mathbf{O} 2]$ extended this result in higher dimension and for $f(u)=|u|^{p-1} u$ $\left(1<p<\frac{N+2}{N-2}\right)$. He also constructs multiple spike solutions. The arguments in $[\mathbf{F W}$, O1, O2] are based on a Lyapunov-Schmidt reduction and rely on the uniqueness and non-degeneracy of the ground state solutions of the autonomous problems :

$$
-\Delta v+V\left(x_{0}\right) v=f(v) \quad \text { in } H^{1}\left(\mathbf{R}^{N}\right) \quad\left(x_{0} \in \mathbf{R}^{N}\right) .
$$

We remark that if we introduce a rescaled (around $x_{0} \in \mathbf{R}^{N}$ ) function $v(y)=u\left(\varepsilon y+x_{0}\right)$, (0.1) becomes $-\Delta v+V\left(x_{0}+\varepsilon y\right) v=f(v)$ and (0.4) appears as a limit as $\varepsilon \rightarrow 0$.

Subsequently reduction methods were also found suitable to find solutions of $(0.1)$ concentrating around possibly degenerate critical points of $V(x)$, when the ground state solutions of the limit problems (0.4) are unique and non-degenerate. In [ABC] Ambrosetti, Badiale and Cingolani consider concentration phenomena at isolated local minima and maxima with polynomial degeneracy and in $[\mathbf{Y Y L}]$ Y. Li deals with $C^{1}$-stable critical points of $V$. See also Grossi $[\mathbf{G r}]$ and Pistoia $[\mathbf{P}]$ for related results. Finally we mention the work of Kang-Wei $[\mathbf{K W}]$ where is establish the existence of positive solutions with any prescribed number of spikes clustering around a given local maximum point of $V(x)$.

We remark that the uniqueness and non-degeneracy of the ground state solutions of (0.4) are, in general, rather difficult to prove. They are known, by means of ODE analysis, only for a rather restricted class of nonlinearities $f(\xi)$ (including $f(\xi)=|\xi|^{p-1} \xi(1<p<$ $\left.\frac{N+2}{N-2}\right)$ ) so far. To attack the existence of positive solutions of (0.1) without assumptions on uniqueness and non-degeneracy, the variational approach, initiated by Rabinowitz $[\mathbf{R}]$, will proved to be successful. In $[\mathbf{R}]$ he proves, by a mountain pass argument, the existence of positive solutions of $(0.1)$, for $\varepsilon>0$ small, whenever

$$
\liminf _{|x| \rightarrow \infty} V(x)>\inf _{x \in \mathbf{R}^{N}} V(x) .
$$


The assumptions on $f(\xi)$ are roughly (f0)-(f4) as given below but no uniqueness nor nondegeneracy conditions on the ground state solutions of (0.4) are required. Later Wang [W] showed that these solutions concentrate at global minimum points of $V(x)$.

In 1996, del Pino and Felmer [DF1] by introducing a penalization approach, so called local mountain pass, managed to handle the case of a, possibly degenerate, local minimum of $V(x)$. More precisely, they assume that an open bounded set $\Lambda \subset \mathbf{R}^{N}$ satisfies

$$
\inf _{x \in \Lambda} V(x)<\min _{x \in \partial \Lambda} V(x)
$$

and they show the existence of a single spike solution concentrating around minimizer of $V(x)$ in $\Lambda$. Very recently they extended their result to the existence of multiple spike solutions in a, possibly degenerate, saddle point setting [DF3]. In [DF3] stronger conditions than in $[\mathbf{D F 1}]$ are required but no assumptions on the uniqueness or the non-degeneracy of solutions of (0.4) are made. As results in between [DF1] and [DF3] we mention [DF2, $\mathrm{Gu}]$.

In the present paper we focus on the existence of solutions of (0.1) concentrating in a given set of local minima of $V(x)$. We introduce new techniques which permit to extend the result of del Pino-Felmer $[\mathbf{D F} 1]$ to a wider class on nonlinearities $f(\xi) \in C^{1}(\mathbf{R}, \mathbf{R})$. In [DF1] the assumptions on $f(\xi)$ are the following :

(f0) $f(\xi) \in C^{1}(\mathbf{R}, \mathbf{R})$.

(f1) $f(\xi)=o(\xi)$ as $\xi \sim 0$.

(f2) For some $s \in\left(1, \frac{N+2}{N-2}\right)$ if $N \geq 3$ and for some $s \in(1, \infty)$ if $N=1,2$

$$
\frac{f(\xi)}{\xi^{s}} \rightarrow 0 \quad \text { as } \xi \rightarrow \infty
$$

(f3) There exists $\mu>2$ such that

$$
0<\mu F(\xi) \leq f(\xi) \xi \text { for all } \xi>0
$$

where

$$
F(\xi)=\int_{0}^{\xi} f(\tau) d \tau .
$$

(f4) The function $\xi \mapsto \frac{f(\xi)}{\xi} ;(0, \infty) \rightarrow \mathbf{R}$ is nondecreasing. Under (f0)-(f3), the functional $I_{\varepsilon}(u)$ corresponding to $(0.1)$

$$
I_{\varepsilon}(u)=\frac{1}{2} \int_{\mathbf{R}^{N}} \varepsilon^{2}|\nabla u|^{2}+V(x) u^{2} d x-\int_{\mathbf{R}^{N}} F(u) d x
$$


has a mountain pass geometry.

In the proofs of [DF1] the assumptions (f3) and (f4) play important roles. (f3) is called the global Ambrosetti-Rabinowitz's condition. It ensures the boundedness of PalaisSmale sequences for $I_{\varepsilon}$. We also remark that (f3) implies that $f(\xi)$ is superlinear, namely that

$$
\frac{f(\xi)}{\xi} \rightarrow \infty \quad \text { as } \xi \rightarrow \infty .
$$

The condition (f4) guarantees the following properties for $I_{\varepsilon}$; for any $u \neq 0$, the real function defined on $(0, \infty) \rightarrow \mathbf{R}$ by $t \mapsto I_{\varepsilon}(t u)$, takes a unique local (hence global) maximum. This enables to make use of the Nehari manifold $\mathcal{M}=\left\{u \in H^{1}\left(\mathbf{R}^{N}\right) \backslash\{0\} ; I_{\varepsilon}^{\prime}(u) u=0\right\}$ and to show that a mountain pass critical point for $I_{\varepsilon}(u)$ is a least energy solution of (0.1).

We will show that the result of [DF1] holds without the assumption (f4). We also introduce a new condition (f5) which can replace (f3) to ensure the boundedness of Cerami sequences and enables to consider asymptotically linear problems. Our main result is the following :

Theorem 0.1. Suppose $N \geq 2$ and assume that $f(\xi)$ satisfies (f0)-(f2) and either (f3) or

(f5) (i) There exists $a \in(0, \infty]$ such that

$$
\frac{f(\xi)}{\xi} \rightarrow a \quad \text { as } \xi \rightarrow \infty
$$

(ii) There exists a constant $D \geq 1$ such that

$$
\widehat{F}(s) \leq D \widehat{F}(t) \quad \text { for all } 0 \leq s \leq t
$$

where

$$
\widehat{F}(\xi)=\frac{1}{2} f(\xi) \xi-F(\xi)
$$

Let $\Lambda \subset \mathbf{R}^{N}$ be a bounded open set satisfying

$$
\inf _{x \in \Lambda} V(x)<\min _{x \in \partial \Lambda} V(x)
$$

and, in case $a<\infty$ in (f5),

$$
\inf _{x \in \Lambda} V(x)<a .
$$

Then there exists an $\varepsilon_{0}>0$ such that for any $\varepsilon \in\left(0, \varepsilon_{0}\right],(0.1)$ has a solution $u_{\varepsilon}(x)$ satisfying

$1^{\circ} u_{\varepsilon}(x)$ has unique local maximum (hence global maximum) in $\mathbf{R}^{N}$ at $x_{\varepsilon} \in \Lambda$. 
$2^{\circ} V\left(x_{\varepsilon}\right) \rightarrow \inf _{x \in \Lambda} V(x)$.

$3^{\circ}$ There exist constants $C_{1}, C_{2}>0$ such that

$$
u_{\varepsilon}(x) \leq C_{1} \exp \left(-C_{2} \frac{\left|x-x_{\varepsilon}\right|}{\varepsilon}\right) \quad \text { for } x \in \mathbf{R}^{N} .
$$

In Section 1, we will see that (f4) implies (f5) with $D=1$. Thus as a special case of Theorem 0.1 we have

Theorem 0.2. Assume that $f(\xi)$ satisfies (f0)-(f2) and either (f3) or (f4). Then the conclusion of Theorem 0.1 holds.

Remark 0.3. (i) In [DF1] del Pino-Felmer showed the existence of a solution $u_{\varepsilon}(x)$ satisfying $1^{\circ}-3^{\circ}$ in the statement of Theorem 0.1 under the conditions (f0)-(f2) and both (f3) and (f4). Thus our theorem generalizes their result. In Section 1 we give some examples of nonlinearities that our result now permit to consider.

(ii) When $N=1$, the existence of solutions concentrating in a bounded open set $\Lambda \subset \mathbf{R}$ satisfying (0.7) can be shown under weaker conditions, namely, under (f0), (f1) and :

(f6) There exists $\xi_{0}>0$ such that

$$
\begin{aligned}
& -\frac{\sigma}{2} \xi^{2}+F(\xi)<0 \quad \text { for } \xi \in\left(0, \xi_{0}\right) \\
& -\frac{\sigma}{2} \xi_{0}^{2}+F\left(\xi_{0}\right)=0 \\
& -\sigma \xi_{0}+f\left(\xi_{0}\right)>0
\end{aligned}
$$

where $\sigma=\inf _{x \in \Lambda} V(x)$.

For the proof we follow [DFT] where a broken geodesic type argument is developed for 1-dimensional nonlinear Schrödinger equations. See also $[\mathbf{N a T}]$ for a related argument for 1-dimensional spatially inhomogeneous phase transition problems.

Concerning the removal of (f4) one of the keys of our proof is to use our recent work [JT2] on autonomous nonlinear scalar field equations in $\mathbf{R}^{N}$, where we show that under the same conditions which guarantee the existence of a least energy solution (see [BL], $[\mathbf{B G K}])$ these solutions possess a mountain pass characterization. Without assuming (f4) such property do not hold for $I_{\varepsilon}(u)$, and in particular our solutions $u_{\varepsilon}(x)$ may not be least energy solutions. However, to prove Theorem 0.1 we just use the mountain pass characterization on the limit equations (0.4).

To get Theorem 0.1 without assuming (f3) we have to overcome the problems of proving the boundedness of Palais-Smale sequences (or at least of Cerami sequences). For 
this we take advantage of some techniques introduced by Jeanjean $[\mathbf{J}]$ and further extended in Jeanjean-Tanaka [JT1]. Our condition (f5) do not force $f(\xi)$ to be superlinear and we manage to handle cases where it is asymptotically linear. To our knowledge there does not exist other existence result for such nonlinearities (without assumptions on uniqueness and non-degeneracy of solutions of (0.4)).

To end this introduction we mention that equations of the form of (0.1) set on a bounded domain of $\mathbf{R}^{N}$ under Dirichlet or Neumann boundary conditions have also drawn considerable attention these last years, starting with the works of Ni-Takagi [NT1, NT2] and Ni-Wei $[\mathbf{N W}]$. Motivated by mathematical models in biology, the existence of single and multiple spike solutions is studied. We suspect that the techniques we develop in this paper could induce new progress in this direction.

The proof of Theorem 0.1 consists of several steps. In Section 1, influenced by the work of del Pino-Felmer [DF1], we introduce a modified functional for any $\varepsilon>0$ and show it has a mountain pass geometry. Next, in Section 2, we study the boundedness of Cerami sequences for the modified functionals. We give two types of boundedness results; one when $\varepsilon>0$ is fixed, the other one to obtain uniform boundedness when $\varepsilon$ goes to 0. In Section 3 , we study the behavior of bounded Cerami sequences. We develop parameter-dependent concentration-compactness type argument, which may be regarded as a generalization of Proposition 2.2 of Gui $[\mathbf{G u}]$, and we believe that it could be useful in other situations. In Section 4, we study the limit equations (0.4). Finally in Section 5, we end the proof of Theorem 0.1, showing that the critical points of the modified functionals satisfies, after a rescaling, the original problem (0.1). 


\section{Setting the modified problems}

In this section we give some preliminaries for the proof of Theorem 0.1. Since we seek positive solutions, we can assume that

$$
f(\xi)=0 \quad \text { for all } \xi \leq 0
$$

First we summarize some basic properties of $f(\xi)$.

Lemma 1.1. Assume (f0)-(f2). Then

(i) For any $\delta>0$ there exists $C_{\delta}>0$ such that

$$
|f(\xi)| \leq \delta|\xi|+C_{\delta}|\xi|^{s} \quad \text { for all } \xi \in \mathbf{R}
$$

(ii) If (f3) is satisfied, $f(\xi) \geq 0$ for all $\xi \geq 0$.

(iii) If (f5) is satisfied, $f(\xi) \geq 0, \widehat{F}(\xi) \geq 0, \frac{d}{d \xi}\left(\frac{F(\xi)}{\xi^{2}}\right) \geq 0$ for all $\xi \geq 0$.

(iv) If $f(\xi)$ satisfies (f4), then (f5) holds with $D=1$.

Proof. (i), (ii) are trivial. To show (iii) we set $s=0$ in (0.6). We get

$$
\widehat{F}(t) \geq 0 \quad \text { for all } t \geq 0
$$

Thus

$$
\frac{d}{d t}\left(\frac{F(t)}{t^{2}}\right)=\frac{2 \widehat{F}(t)}{t^{3}} \geq 0
$$

(1.4) implies under (f1) that

$$
\frac{F(t)}{t^{2}} \geq \lim _{t \rightarrow 0} \frac{F(t)}{t^{2}}=0 \quad \text { for all } t>0
$$

Finally combining (1.3) and (1.5), we have

$$
\frac{1}{2} f(t) t=\widehat{F}(t)+F(t) \geq 0 \quad \text { for all } t \geq 0 .
$$

(iv) For $0<s<t$ we have

$$
\begin{aligned}
\widehat{F}(t)-\widehat{F}(s) & =\frac{1}{2}(f(t) t-f(s) s)-(F(t)-F(s)) \\
& =\int_{0}^{t} \frac{f(t)}{t} \tau d \tau-\int_{0}^{s} \frac{f(s)}{s} \tau d \tau-\int_{s}^{t} \frac{f(\tau)}{\tau} \tau d \tau \\
& =\int_{s}^{t}\left(\frac{f(t)}{t}-\frac{f(\tau)}{\tau}\right) \tau d \tau+\int_{0}^{s}\left(\frac{f(t)}{t}-\frac{f(s)}{s}\right) \tau d \tau \\
& \geq 0
\end{aligned}
$$


Thus (f5) with $D=1$ follows.

Here are some examples of nonlinearities which satisfies our conditions.

\section{Examples.}

(i) $f(\xi)=\xi \log (1+\xi)$ satisfies (f0)-(f2), (f5) with $a=\infty, D=1$ but not (f3).

(ii) $f(\xi)=\frac{\xi^{2}}{1+k \xi}(k>0)$ satisfies (f0)-(f2), (f5) with $a=\frac{1}{k}, D=1$.

(iii) For an example satisfying (f0)-(f2), (f5) with $D>1$, we claim that we can re-construct $f(\xi)$ from $\widehat{F}(\xi)$. Indeed under (f1), (f5) it holds :

(i) $\widehat{F}(\xi)=O\left(\xi^{3}\right)$ at $\xi=0$.

(ii) $\widehat{F}(\xi) \geq 0$ for all $\xi>0$.

(iii) (0.6) holds if and only if

$$
\sup _{0<s<t} \frac{\widehat{F}(s)}{\widehat{F}(t)}<\infty .
$$

(Here we regard $\widehat{F}(s)=0$ if $\widehat{F}(t)=0)$.

Now for a given function $\widehat{F}(\xi) \in C([0, \infty), \mathbf{R})$ satisfying (1.6)-(1.8), we set

$$
F(\xi)=2 \xi^{2} \int_{0}^{\xi} \frac{\widehat{F}(\tau)}{\tau^{3}} d \tau
$$

We can easily check that $\frac{1}{2} F^{\prime}(\xi) \xi-F(\xi)$ equals to the given $\widehat{F}(\xi)$. Thus for a given $\widehat{F}(\xi) \in$ $C([0, \infty), \mathbf{R})$ satisfying $(1.6)-(1.8)$, we can re-construct $f(\xi)$. For example for a function satisfying (1.6), (1.7), $f^{\prime}(\xi) \geq 0$ near $\xi=0$ and $0<\liminf _{\xi \rightarrow \infty} \widehat{F}(\xi) \leq \limsup _{\xi \rightarrow \infty} \widehat{F}(\xi)<$ $\infty$, we can find $f(\xi)$ which satisfies (f0)-(f2), (f5).

\section{(a) Modification of the nonlinearity $f(\xi)$}

To find a solution $u_{\varepsilon}(x)$ concentrating in a given set $\Lambda$, we modify the nonlinearity $f(\xi)$. Here we follow an approach inspired by del Pino-Felmer [DF1].

Let $f(\xi)$ be a function satisfying (f0)-(f2) and $V_{0}<a=\lim _{\xi \rightarrow \infty} \frac{f(\xi)}{\xi} \in(0, \infty]$. We choose a small number $\nu \in\left(0, \frac{V_{0}}{2}\right)$ and we set

$$
\underline{f}(\xi)= \begin{cases}\min \{f(\xi), \nu \xi\} & \text { for } \xi \geq 0 \\ 0 & \text { for } \xi<0 .\end{cases}
$$

By (f1) we can see that there exists a small $r_{\nu}>0$ such that

$$
\underline{f}(\xi)=f(\xi) \quad \text { for }|\xi| \leq r_{\nu} .
$$

Moreover there holds

$$
\begin{aligned}
& \underline{f}(\xi)=\nu \xi \quad \text { for large } \xi \geq 0, \\
& \underline{f}(\xi)=0 \quad \text { for } \xi \leq 0 .
\end{aligned}
$$


For technical reasons, we choose $\nu$ in the following way :

$1^{\circ}$ Under (f3), we choose $\nu>0$ so that

$$
\frac{\nu}{2 V_{0}}<\frac{1}{2}-\frac{1}{\mu}
$$

$2^{\circ}$ Under (f5), we choose $\nu \in\left(0, \frac{V_{0}}{2}\right)$ so that $\nu$ is a regular value of $\xi \mapsto \frac{f(\xi)}{\xi}$ : $(0, \infty) \rightarrow \mathbf{R}$. Since $\lim _{\xi \rightarrow 0} \frac{f(\xi)}{\xi}=0$ and $\lim _{\xi \rightarrow \infty} \frac{f(\xi)}{\xi}=a>V_{0}>\nu$, if $\nu$ is a regular value of $\frac{f(\xi)}{\xi}$, we can see that

$$
k_{\nu} \equiv \#\{\xi \in(0, \infty) ; f(\xi)=\nu \xi\}<\infty .
$$

Next, let $\Lambda \subset \mathbf{R}^{N}$ be a bounded open set satisfying (0.7). We may assume that the boundary $\partial \Lambda$ is smooth. We choose an open subset $\Lambda^{\prime} \subset \Lambda$ with a smooth boundary $\partial \Lambda^{\prime}$ and a function $\chi(x) \in C^{\infty}\left(\mathbf{R}^{N}, \mathbf{R}\right)$ such that

$$
\begin{array}{ll}
\inf _{x \in \Lambda \backslash \Lambda^{\prime}} V(x)>\inf _{x \in \Lambda} V(x), \\
\min _{x \in \partial \Lambda^{\prime}} V(x)>\inf _{x \in \Lambda^{\prime}} V(x)=\inf _{x \in \Lambda} V(x), \\
\chi(x)=1 \quad \text { for } x \in \Lambda^{\prime}, \\
\chi(x) \in(0,1) & \text { for } x \in \Lambda \backslash \overline{\Lambda^{\prime}}, \\
\chi(x)=0 & \text { for } x \in \mathbf{R}^{N} \backslash \Lambda .
\end{array}
$$

In what follows we assume, without loss of generality, that

$$
0 \in \Lambda^{\prime} \quad \text { and } \quad V(0)=\inf _{x \in \Lambda} V(x) .
$$

Finally we define

$$
g(x, \xi)=\chi(x) f(\xi)+(1-\chi(x)) \underline{f}(\xi) \quad \text { for }(x, \xi) \in \mathbf{R}^{N} \times \mathbf{R}
$$

and we write $\underline{F}(\xi)=\int_{0}^{\xi} \underline{f}(\tau) d \tau, G(x, \xi)=\int_{0}^{\xi} g(x, \tau) d \tau=\chi(x) F(\xi)+(1-\chi(x)) \underline{F}(\xi)$.

From now on we try to find a solution of the following problem :

$$
-\varepsilon^{2} \Delta u+V(x) u=g(x, u) \text { in } \mathbf{R}^{N} .
$$

We will find a solution $u_{\varepsilon}(x)$ of (1.14) via a mountain pass argument and besides other properties we will show that the mountain pass solution $u_{\varepsilon}(x)$ satisfies for small $\varepsilon>0$

$$
\left|u_{\varepsilon}(x)\right| \leq r_{\nu} \quad \text { for } x \in \mathbf{R}^{N} \backslash \Lambda^{\prime},
$$

that is, $u_{\varepsilon}(x)$ also solves the original problem $(0.1)$.

We give some fundamental properties of $\underline{f}(\xi)$. 
Lemma 1.2. (i) $\underline{f}(\xi)=0, \underline{F}(\xi)=0$ for all $\xi \leq 0$.

(ii) $\underline{f}(\xi) \leq \nu \xi, \underline{F}(\xi) \leq F(\xi)$ for $\xi \geq 0$.

(iii) $\underline{f}(\xi) \leq f(\xi)$ for $\xi \geq 0$.

(iv) If $f(\xi)$ satisfies either (f3) or (f5), then it holds that $f(\xi) \geq 0$ for all $\xi \in \mathbf{R}$.

(v) If $f(\xi)$ satisfies (f5), then $\underline{f}(\xi)$ also satisfies (f5). In particular, $\underline{F}(\xi) \geq 0$ for all $\xi \geq 0$.

Proof. (i)-(iv) are trivial from the definition of $\underline{f}(\xi)$. For $(\mathrm{v})$, we recall that $\nu$ is taken so that (1.11) holds. First we consider the case

$$
\underline{f}(\xi)=f(\xi) \text { in }[s, t] .
$$

Then

$$
\begin{aligned}
\underline{\widehat{F}}(t) & \geq \frac{1}{2} f(t) t-\int_{0}^{t} \underline{f}(\tau) d \tau=\frac{1}{2} f(t) t-F(t)+F(s)-\underline{F}(s) \\
& =\widehat{F}(t)+F(s)-\underline{F}(s) .
\end{aligned}
$$

Since $f(\xi)$ satisfies $(\mathrm{f} 5)$ and $F(s)-\underline{F}(s) \geq 0$, we have

$$
\begin{aligned}
& \geq \frac{1}{D} \widehat{F}(s)+\frac{1}{D}(F(s)-\underline{F}(s))=\frac{1}{D}\left(\frac{1}{2} f(s) s-\underline{F}(s)\right) \\
& =\frac{1}{D} \underline{\widehat{F}}(s) .
\end{aligned}
$$

Next if

$$
\underline{f}(\xi)=\nu \xi \quad \text { in }[s, t]
$$

we have

$$
\begin{aligned}
\underline{\widehat{F}}(t) & =\frac{1}{2} \nu t^{2}-\int_{s}^{t} \nu \tau d \tau-\int_{0}^{s} \underline{f}(\tau) d \tau \\
& =\frac{1}{2} \nu s^{2}-\int_{0}^{s} \underline{f}(\tau) d \tau=\underline{\widehat{F}}(s) \geq \frac{1}{D} \underline{\widehat{F}}(s) .
\end{aligned}
$$

Thus in the general case, we can find a sequence $s=\alpha_{0}<\alpha_{1}<\alpha_{2}<\cdots<\alpha_{n-1}<\alpha_{n}=t$ such that $f\left(\alpha_{j}\right)=\nu \alpha_{j}(j=1,2, \cdots, n-1)$ and in each interval $\left[\alpha_{j}, \alpha_{j+1}\right],(1.16)$ or $(1.17)$ holds. We use the above fact repeatedly and obtain

$$
\underline{\widehat{F}}(s) \leq D \underline{\widehat{F}}\left(\alpha_{1}\right) \leq D^{2} \underline{\widehat{F}}\left(\alpha_{2}\right) \leq \cdots \leq D^{n-1} \widehat{F}\left(\alpha_{n-1}\right) \leq D^{n} \underline{\widehat{F}}(t) .
$$

By $(1.11), n$ is bounded by $k_{\nu}$ and we get

$$
\widehat{F}(s) \leq D^{k_{\nu}} \widehat{F}(t) \text { for all } 0 \leq s \leq t
$$

Replacing $D$ with $D^{k_{\nu}}$, this is the desired result. 
Corollary 1.3. (i) $g(x, \xi) \leq f(\xi), G(x, \xi) \leq F(\xi)$ for all $(x, \xi) \in \mathbf{R}^{N} \times \mathbf{R}$.

(ii) $g(x, \xi)=f(\xi)$ if $|\xi|<r_{\nu}$.

(iii) For any $\delta>0$ there exists $C_{\delta}>0$ such that

$$
|g(x, \xi)| \leq \delta|\xi|+C_{\delta}|\xi|^{s+1} \quad \text { for all }(x, \xi) \in \mathbf{R}^{N} \times \mathbf{R}
$$

(iv) If $f(\xi)$ satisfies (f5)-(ii), then $g(x, \xi)$ also satisfies

$$
\widehat{G}(x, s) \leq D^{k_{\nu}} \widehat{G}(x, t) \quad \text { for } 0 \leq s \leq t
$$

where $\widehat{G}(x, \xi)=\frac{1}{2} g(x, \xi) \xi-G(x, \xi), D \geq 1$ is given in (f5)-(ii) and $k_{\nu}$ is given in (1.11).

Proof. By the definition of $g(x, \xi)$ in (1.13), (i)-(iv) follow easily from Lemma 1.2.

(b) The modified functional

Introducing the re-scaled function $v(y)=u(\varepsilon y)$ we can rewrite (1.14) as

$$
-\Delta v+V(\varepsilon y) v=g(\varepsilon y, v) \text { in } \mathbf{R}^{N} .
$$

We shall mainly deal with (1.18) instead of (1.14).

The functional corresponding to (1.18) is

$$
J_{\varepsilon}(v)=\frac{1}{2} \int_{\mathbf{R}^{N}}|\nabla v|^{2}+V(\varepsilon y) v^{2} d y-\int_{\mathbf{R}^{N}} G(\varepsilon y, v) d y .
$$

We consider $J_{\varepsilon}(v)$ on the following function space :

$$
H_{\varepsilon}=\left\{v \in H^{1}\left(\mathbf{R}^{N}\right) ; \int_{\mathbf{R}^{N}} V(\varepsilon y) v^{2} d y<\infty\right\}
$$

equipped with norm

$$
\|v\|_{H_{\varepsilon}}^{2}=\int_{\mathbf{R}^{N}}|\nabla v|^{2}+V(\varepsilon y) v^{2} d y .
$$

We shall make use the following notation :

$$
\begin{aligned}
\|u\|_{r}^{r} & =\int_{\mathbf{R}^{N}}|u|^{r} d y \quad \text { for } r \in[1, \infty), \\
\|u\|_{\infty} & =\underset{x \in \mathbf{R}^{N}}{\operatorname{ess} \sup }|u(x)|, \\
\|u\|_{H^{1}\left(\mathbf{R}^{N}\right)}^{2} & =\int_{\mathbf{R}^{N}}|\nabla v|^{2}+V_{0} v^{2} d y .
\end{aligned}
$$


Here $V_{0}>0$ is the constant appearing in (0.2) and thus $\|\cdot\|_{H^{1}\left(\mathbf{R}^{N}\right)}$ is equivalent to the standard $H^{1}\left(\mathbf{R}^{N}\right)$-norm. Since

$$
\|v\|_{H^{1}\left(\mathbf{R}^{N}\right)} \leq\|v\|_{H_{\varepsilon}}
$$

we have $H_{\varepsilon} \subset H^{1}\left(\mathbf{R}^{N}\right)$ and $H_{\varepsilon}$ can be embedded into $L^{r}\left(\mathbf{R}^{N}\right)\left(2 \leq r \leq \frac{2 N}{N-2}\right.$ for $N \geq 3$, $2 \leq r<\infty$ for $N=2$ ) continuously, i.e., there exists $C_{r}^{\prime}>0$ such that

$$
\|v\|_{r} \leq C_{r}^{\prime}\|v\|_{H^{1}\left(\mathbf{R}^{N}\right)} \text { for all } v .
$$

Proposition 1.4. $J_{\varepsilon}(v) \in C^{1}\left(H_{\varepsilon}, \mathbf{R}\right)$ and it has a Mountain Pass Geometry that is uniform with respect to $\varepsilon$ in the following sense :

$1^{\circ} J_{\varepsilon}(0)=0$.

$2^{\circ}$ There are constants $\rho_{0}>0$ and $\delta_{0}>0$ independent of $\varepsilon \in(0,1]$ such that

$$
J_{\varepsilon}(v) \geq \delta_{0} \quad \text { for all }\|v\|_{H^{1}\left(\mathbf{R}^{N}\right)}=\rho_{0}
$$

and

$$
J_{\varepsilon}(v)>0 \quad \text { for all } 0<\|v\|_{H^{1}\left(\mathbf{R}^{N}\right)} \leq \rho_{0} .
$$

$3^{\circ}$ There is a $v_{0}(x) \in C_{0}^{\infty}\left(\mathbf{R}^{N}\right)$ and $\varepsilon_{0}>0$ such that

$$
J_{\varepsilon}\left(v_{0}\right)<0 \quad \text { for all } \varepsilon \in\left(0, \varepsilon_{0}\right]
$$

Proof. Since $J_{\varepsilon}(v)=\frac{1}{2}\|v\|_{H_{\varepsilon}}^{2}-\int_{\mathbf{R}^{N}} G(\varepsilon y, v) d y$, it is clear that $J_{\varepsilon}(v) \in C^{1}\left(H_{\varepsilon}, \mathbf{R}\right) .1^{\circ}$ is also trivial. To show $2^{\circ}$, using (1.2), (1.19), (1.20), we compute

$$
\begin{aligned}
J_{\varepsilon}(v) & =\frac{1}{2}\|v\|_{H_{\varepsilon}}^{2}-\int_{\mathbf{R}^{N}} \chi(\varepsilon y) F(v)+(1-\chi(\varepsilon y)) \underline{F}(v) d y \\
& \geq \frac{1}{2}\|v\|_{H_{\varepsilon}}^{2}-\int_{\mathbf{R}^{N}} F(v) d y \\
& \geq \frac{1}{2}\|v\|_{H^{1}\left(\mathbf{R}^{N}\right)}^{2}-\frac{V_{0}}{4}\|v\|_{2}^{2}-C_{V_{0} / 2}\|v\|_{s+1}^{s+1} \\
& \geq \frac{1}{4}\|v\|_{H^{1}\left(\mathbf{R}^{N}\right)}^{2}-C_{V_{0} / 2} C_{s+1}^{\prime}\|v\|_{H^{1}\left(\mathbf{R}^{N}\right)}^{s+1} .
\end{aligned}
$$

Thus we can find constants $\rho_{0}, \delta_{0}>0$ such that the statement $2^{\circ}$ holds.

To show $3^{\circ}$, we choose $v_{0} \in C_{0}^{\infty}\left(\mathbf{R}^{N}\right)$ such that

$$
\frac{1}{2} \int_{\mathbf{R}^{N}}\left|\nabla v_{0}\right|^{2}+V(0) v_{0}^{2} d y-\int_{\mathbf{R}^{N}} F\left(v_{0}\right) d y<0 .
$$


Recall that $V(0)<\lim _{z \rightarrow \infty} \frac{f(z)}{z}$. Then the existence of such $v_{0} \in C_{0}^{\infty}\left(\mathbf{R}^{N}\right)$ follows from Proposition 4.2, where it is proved that $v \rightarrow \frac{1}{2} \int_{\mathbf{R}^{N}}\left|\nabla v_{0}\right|^{2}+V(0) v_{0}^{2} d y-\int_{\mathbf{R}^{N}} F\left(v_{0}\right) d y$ has a mountain pass geometry. Since we are assuming $0 \in \Lambda^{\prime}$, we observe that

$$
J_{\varepsilon}\left(v_{0}\right) \rightarrow \frac{1}{2} \int_{\mathbf{R}^{N}}\left|\nabla v_{0}\right|^{2}+V(0) v_{0}^{2} d y-\int_{\mathbf{R}^{N}} F\left(v_{0}\right) d y<0 \quad \text { as } \varepsilon \rightarrow 0 .
$$

Thus we get $3^{\circ}$ for sufficiently small $\varepsilon>0$

By Proposition 1.4, we can define the mountain pass value. For $\varepsilon \in\left(0, \varepsilon_{0}\right]$ we set

$$
\begin{aligned}
b_{\varepsilon} & =\inf _{\gamma \in \Gamma_{\varepsilon}} \max _{t \in[0,1]} J_{\varepsilon}(\gamma(t)), \\
\Gamma_{\varepsilon} & =\left\{\gamma \in C\left([0,1], H_{\varepsilon}\right) ; \gamma(0)=0, J_{\varepsilon}(\gamma(1))<0\right\} .
\end{aligned}
$$

In what follows, we will show that for $\varepsilon$ small, $b_{\varepsilon}$ is a critical value of $J_{\varepsilon}(v)$ and the corresponding critical point has - after re-scaling — exactly one peak in $\Lambda$.

By the above Proposition 1.4, we have the following a priori bound for the mountain pass value $b_{\varepsilon}$.

Corollary 1.5. There are constants $m_{1}, m_{2}>0$ such that for $\varepsilon \in\left(0, \varepsilon_{0}\right]$

$$
m_{1} \leq b_{\varepsilon} \leq m_{2}
$$

Proof. Since

$$
\gamma([0,1]) \cap\left\{v \in H_{\varepsilon} ;\|v\|_{H^{1}\left(\mathbf{R}^{N}\right)}=\rho_{0}\right\} \neq \emptyset
$$

for any $\gamma \in \Gamma_{\varepsilon}$, by Proposition 1.4, we have

$$
\max _{t \in[0,1]} J_{\varepsilon}(\gamma(t)) \geq \inf _{\|v\|_{H^{1}\left(\mathbf{R}^{N}\right)}=\rho_{0}} J_{\varepsilon}(v) \geq \delta_{0}
$$

On the other hand, taking a path $\gamma(t)=t v_{0}$, where $v_{0} \in C_{0}^{\infty}\left(\mathbf{R}^{N}\right)$ is given in Proposition 1.4, we have

$$
b_{\varepsilon} \leq \sup _{\varepsilon \in\left(0, \varepsilon_{0}\right]} \max _{t \in[0,1]} J_{\varepsilon}\left(\gamma_{0}(t)\right) \equiv m_{2}
$$

Thus we get (1.23) with $m_{1}=\delta_{0}$ and $m_{2}$ given in the above formula.

In the following sections, we will discuss the boundedness of Cerami sequence corresponding to $b_{\varepsilon}$. We will also discuss "uniform boundedness" with respect to $\varepsilon \in\left(0, \varepsilon_{0}\right]$. 


\section{Boundedness of Cerami sequences}

From Proposition 1.4 and Ekeland's principle, for any $\varepsilon \in\left(0, \varepsilon_{0}\right]$ there exists a Cerami sequence $\left(v_{j}\right)_{j=1}^{\infty} \subset H_{\varepsilon}$ at level $b_{\varepsilon}$ :

$$
\begin{aligned}
& J_{\varepsilon}\left(v_{j}\right) \rightarrow b_{\varepsilon}, \\
& \left(1+\left\|v_{j}\right\|_{H_{\varepsilon}}\right)\left\|J_{\varepsilon}^{\prime}\left(v_{j}\right)\right\|_{H_{\varepsilon}^{*}} \rightarrow 0 \quad \text { as } j \rightarrow \infty .
\end{aligned}
$$

We will show under the assumptions (f3) or (f5) that $\left(v_{j}\right)_{j=1}^{\infty}$ is bounded in $H_{\varepsilon}$ and has a convergent subsequence. Thus $J_{\varepsilon}(v)$ has a critical point $v_{\varepsilon}$ satisfying

$$
\begin{aligned}
& J_{\varepsilon}^{\prime}\left(v_{\varepsilon}\right)=0, \\
& J_{\varepsilon}\left(v_{\varepsilon}\right)=b_{\varepsilon} .
\end{aligned}
$$

Also we show that $\left(v_{\varepsilon}\right)$ is bounded in the sense that

$$
\limsup _{\varepsilon \rightarrow 0}\left\|v_{\varepsilon}\right\|_{H_{\varepsilon}}<\infty
$$

This type of boundedness is important in our argument. More precisely we show :

Proposition 2.1. Assume that $f(\xi)$ satisfies (f0)-(f2) and either (f3) or (f5). Then there exists $\varepsilon_{1} \in\left(0, \varepsilon_{0}\right]$ such that for any $\varepsilon \in\left(0, \varepsilon_{1}\right]$ and for any sequence $\left(v_{j}\right) \subset H_{\varepsilon}$ satisfying

$$
\begin{aligned}
& J_{\varepsilon}\left(v_{j}\right) \rightarrow c>0, \\
& \left(1+\left\|v_{j}\right\|_{H_{\varepsilon}}\right)\left\|J_{\varepsilon}^{\prime}\left(v_{j}\right)\right\|_{H_{\varepsilon}^{*}} \rightarrow 0 \quad \text { as } j \rightarrow \infty
\end{aligned}
$$

for some $c>0$, we have

(i) $\left\|v_{j}\right\|_{H_{\varepsilon}}$ is bounded as $j \rightarrow \infty$.

(ii) There exist a subsequence $j_{k}$ and $v_{0} \in H_{\varepsilon}$ such that

$$
v_{j_{k}} \rightarrow v_{0} \quad \text { strongly in } H_{\varepsilon} \text {. }
$$

Proposition 2.2. Assume that $f(\xi)$ satisfies (f0)-(f2) and either (f3) or (f5). Suppose that a sequence $\left(v_{\varepsilon}\right)_{\varepsilon \in\left(0, \varepsilon_{1}\right]}$ satisfies

$$
\begin{aligned}
& v_{\varepsilon} \in H_{\varepsilon} \\
& J_{\varepsilon}\left(v_{\varepsilon}\right) \in\left[m_{1}, m_{2}\right] \quad \text { for all } \varepsilon \in\left(0, \varepsilon_{1}\right], \\
& \left(1+\left\|v_{\varepsilon}\right\|_{H_{\varepsilon}}\right)\left\|J_{\varepsilon}^{\prime}\left(v_{\varepsilon}\right)\right\|_{H_{\varepsilon}^{*}} \rightarrow 0 \quad \text { as } \varepsilon \rightarrow 0
\end{aligned}
$$


for $m_{2}>m_{1}>0$. Then (2.1) holds.

Since the proofs of Propositions 2.1 (i) and 2.2 have many similar points, we mainly deal with Proposition 2.2. First we prove it under (f0)-(f2) and (f3). In what follows we write $\chi_{\varepsilon}(y)=\chi(\varepsilon y)$.

Proof of Proposition 2.2 under condition (f3). This is quite standard. Let $\left(v_{\varepsilon}\right)$ satisfy (2.4) and (2.5). From (2.4) we have

$$
\frac{1}{2}\left\|v_{\varepsilon}\right\|_{H_{\varepsilon}}^{2}-\int_{\mathbf{R}^{N}}(1-\chi(\varepsilon y)) \underline{F}\left(v_{\varepsilon}\right)+\chi(\varepsilon y) F\left(v_{\varepsilon}\right) d y \leq m_{2} .
$$

From (2.5) it follows that $\left|J_{\varepsilon}^{\prime}\left(v_{\varepsilon}\right) v_{\varepsilon}\right| \leq\left\|J_{\varepsilon}^{\prime}\left(v_{\varepsilon}\right)\right\|_{H_{\varepsilon}^{*}}\left\|v_{\varepsilon}\right\|_{H_{\varepsilon}} \leq 1$ for small $\varepsilon$, i.e.,

$$
\left|\left\|v_{\varepsilon}\right\|_{H_{\varepsilon}}^{2}-\int_{\mathbf{R}^{N}}(1-\chi(\varepsilon y)) \underline{f}\left(v_{\varepsilon}\right) v_{\varepsilon}+\chi(\varepsilon y) f\left(v_{\varepsilon}\right) v_{\varepsilon} d y\right| \leq 1 .
$$

Thus computing (2.6) - $\frac{1}{\mu}(2.7)$ and using (f3), we get

$$
\left(\frac{1}{2}-\frac{1}{\mu}\right)\left\|v_{\varepsilon}\right\|_{H_{\varepsilon}}^{2} \leq \int_{\mathbf{R}^{N}}(1-\chi(\varepsilon y))\left(\underline{F}\left(v_{\varepsilon}\right)-\frac{1}{\mu} \underline{f}\left(v_{\varepsilon}\right) v_{\varepsilon}\right) d y+m_{2}+\frac{1}{\mu} .
$$

Recalling that $\xi \underline{f}(\xi) \geq 0$ for all $\xi$, it leads to

$$
\left(\frac{1}{2}-\frac{1}{\mu}\right)\left\|v_{\varepsilon}\right\|_{H_{\varepsilon}}^{2} \leq \int_{\mathbf{R}^{N}}(1-\chi(\varepsilon y)) \underline{F}\left(v_{\varepsilon}\right) d y+m_{2}+\frac{1}{\mu} .
$$

By Lemma 1.2 (ii), we have $\underline{F}(\xi) \leq \frac{1}{2} \nu|\xi|^{2}$ for all $\xi \in \mathbf{R}$. Thus

$$
\int_{\mathbf{R}^{N}}(1-\chi(\varepsilon y)) \underline{F}\left(v_{\varepsilon}\right) d y \leq \frac{1}{2} \nu\left\|v_{\varepsilon}\right\|_{2}^{2} \leq \frac{\nu}{2 V_{0}}\left\|v_{\varepsilon}\right\|_{H_{\varepsilon}}^{2} .
$$

Therefore

$$
\left(\frac{1}{2}-\frac{1}{\mu}\right)\left\|v_{\varepsilon}\right\|_{H_{\varepsilon}}^{2} \leq \frac{\nu}{2 V_{0}}\left\|v_{\varepsilon}\right\|_{H_{\varepsilon}}^{2}+m_{2}+\frac{1}{\mu} .
$$

By our choice (1.10) of $\nu$, we can see that $\left\|v_{\varepsilon}\right\|_{H_{\varepsilon}}$ is bounded as $\varepsilon \rightarrow 0$.

For the proof of Proposition 2.2 under (f5), we use ideas from $[\mathbf{J}]$ and $[\mathbf{J T 1}]$.

Proof of Proposition 2.2 under condition (f5). Following the argument in $[\mathbf{J}]$ and $[\mathbf{J T 1}]$, we argue indirectly and assume that $\lim _{\sup _{\varepsilon \rightarrow 0}}\left\|v_{\varepsilon}\right\|_{H_{\varepsilon}}=\infty$. We take a subsequence $\varepsilon_{j} \rightarrow 0$ such that $\left\|v_{\varepsilon_{j}}\right\|_{H_{\varepsilon_{j}}} \rightarrow \infty$. For simplicity of notation, we write just $\varepsilon$ instead of $\varepsilon_{j}$. 
We set $w_{\varepsilon}=\frac{v_{\varepsilon}}{\left\|v_{\varepsilon}\right\|_{H_{\varepsilon}}}$. Clearly $\left\|w_{\varepsilon}\right\|_{H^{1}\left(\mathbf{R}^{N}\right)} \leq\left\|w_{\varepsilon}\right\|_{H_{\varepsilon}}=1$ and since $\chi_{\varepsilon}$ is uniformly bounded in $C^{1}$ there exists $C_{1}>0$ independent of $\varepsilon>0$ such that

$$
\left\|\chi_{\varepsilon} w_{\varepsilon}\right\|_{H^{1}\left(\mathbf{R}^{N}\right)} \leq C_{1}
$$

Also, since $\left\|J_{\varepsilon}^{\prime}\left(v_{\varepsilon}\right)\right\|_{H_{\varepsilon}}{ } \rightarrow 0$ we have

$$
-\Delta w_{\varepsilon}+V(\varepsilon y) w_{\varepsilon}=\chi_{\varepsilon} \frac{f\left(v_{\varepsilon}\right)}{v_{\varepsilon}} w_{\varepsilon}+\left(1-\chi_{\varepsilon}\right) \frac{f\left(v_{\varepsilon}\right)}{v_{\varepsilon}} w_{\varepsilon}+\frac{o(1)}{\left\|v_{\varepsilon}\right\|_{H_{\varepsilon}}} .
$$

Multiplying $(2.9)$ by $w_{\varepsilon}{ }^{-}(y)=\max \left\{-w_{\varepsilon}(y), 0\right\}$ and integrating over $\mathbf{R}^{N}$, it follows that

$$
\left\|w_{\varepsilon}{ }^{-}\right\|_{H_{\varepsilon}}^{2} \rightarrow 0 \quad \text { as } \varepsilon \rightarrow 0
$$

Now we observe that one of the following 2 cases must take place :

Case $1: \limsup _{\varepsilon \rightarrow 0} \sup _{z \in \mathbf{R}^{N}} \int_{B_{1}(z)}\left|\chi_{\varepsilon}(y) w_{\varepsilon}\right|^{2} d y>0$.

Case $2: \lim _{\varepsilon \rightarrow 0} \sup _{z \in \mathbf{R}^{N}} \int_{B_{1}(z)}\left|\chi_{\varepsilon}(y) w_{\varepsilon}\right|^{2} d y=0$.

Here we use the notation :

$$
B_{1}(y)=\left\{z \in \mathbf{R}^{N} ;|z-y|<1\right\} \quad \text { for } y \in \mathbf{R}^{N}
$$

We will show neither Case 1 nor Case 2 takes place and this will provide the desired contradiction.

Step 1 : Case 1 cannot take place under (f5) with $a=\infty$.

Arguing indirectly, we assume that Case 1 occurs. Then, taking a subsequence if necessary, we can find a sequence $\left(y_{\varepsilon}\right) \subset \mathbf{R}^{N}, d>0$ and $x_{0} \in \bar{\Lambda}$ such that

$$
\begin{aligned}
& \int_{B_{1}\left(y_{\varepsilon}\right)}\left|\chi_{\varepsilon} w_{\varepsilon}\right|^{2} d y \rightarrow d>0, \\
& \varepsilon y_{\varepsilon} \rightarrow x_{0} \in \bar{\Lambda}
\end{aligned}
$$

In fact, since Case 1 occurs, the existence of $\left(y_{\varepsilon}\right)$ with (2.11) is clear. Also, it must be $B_{1}\left(y_{\varepsilon}\right) \cap \operatorname{supp} \chi_{\varepsilon} \neq \emptyset$, that is,

$$
\varepsilon y_{\varepsilon} \in N_{\varepsilon}(\Lambda) \equiv\left\{z \in \mathbf{R}^{N} ; \operatorname{dist}(z, \Lambda)<\varepsilon\right\}
$$

Thus we may assume $\varepsilon y_{\varepsilon} \rightarrow x_{0} \in \bar{\Lambda}$. Extracting a subsequence again, there holds

$$
w_{\varepsilon}\left(y+y_{\varepsilon}\right) \rightarrow w_{0}(y) \quad \text { weakly in } H^{1}\left(\mathbf{R}^{N}\right)
$$


Then we have

$$
\left(\chi_{\varepsilon} w_{\varepsilon}\right)\left(y+y_{\varepsilon}\right)=\chi\left(\varepsilon y+\varepsilon y_{\varepsilon}\right) w_{\varepsilon}\left(y+y_{\varepsilon}\right) \rightarrow \chi\left(x_{0}\right) w_{0}(y) \quad \text { weakly in } H^{1}\left(\mathbf{R}^{N}\right) .
$$

By $(2.10)$ and (2.11), we can see $\chi\left(x_{0}\right) \neq 0$ and $w_{0}(y) \geq 0(\not \equiv 0)$. In particular, we can find a set $K \subset \mathbf{R}^{N}$ such that

$$
\text { meas } K>0
$$

and

$$
w_{\varepsilon}\left(y+y_{\varepsilon}\right) \rightarrow w_{0}(y)>0 \quad \text { for } y \in K .
$$

On the other hand, multiplying (2.9) by $w_{\varepsilon}$ and integrating over $\mathbf{R}^{N}$, it follows that

$$
1=\int_{\mathbf{R}^{N}} \chi_{\varepsilon} \frac{f\left(v_{\varepsilon}\right)}{v_{\varepsilon}} w_{\varepsilon}^{2}+\left(1-\chi_{\varepsilon}\right) \frac{f\left(v_{\varepsilon}\right)}{v_{\varepsilon}} w_{\varepsilon}^{2} d y+o(1)
$$

and thus

$$
\limsup _{\varepsilon \rightarrow 0} \int_{\mathbf{R}^{N}} \chi_{\varepsilon} \frac{f\left(v_{\varepsilon}\right)}{v_{\varepsilon}} w_{\varepsilon}^{2} d y \leq 1 .
$$

We can rewrite (2.16) in the following way.

$$
\limsup _{\varepsilon \rightarrow 0} \int_{\mathbf{R}^{N}} \chi\left(\varepsilon y+\varepsilon y_{\varepsilon}\right) \frac{f\left(v_{\varepsilon}\left(y+y_{\varepsilon}\right)\right)}{v_{\varepsilon}\left(y+y_{\varepsilon}\right)} w_{\varepsilon}\left(y+y_{\varepsilon}\right)^{2} d y \leq 1 .
$$

By (2.14) and (2.15), we have $v_{\varepsilon}\left(y+y_{\varepsilon}\right) \rightarrow \infty$ as $\varepsilon \rightarrow 0$ for $y \in K$. Therefore by Fatou's lemma and since $\lim _{\xi \rightarrow \infty} \frac{f(\xi)}{\xi}=a=\infty$ we have

$$
\begin{aligned}
& \int_{\mathbf{R}^{N}} \chi\left(\varepsilon y+\varepsilon y_{\varepsilon}\right) \frac{f\left(v_{\varepsilon}\left(y+y_{\varepsilon}\right)\right)}{v_{\varepsilon}\left(y+y_{\varepsilon}\right)} w_{\varepsilon}\left(y+y_{\varepsilon}\right)^{2} d y \\
& \geq \int_{K} \chi\left(\varepsilon y+\varepsilon y_{\varepsilon}\right) \frac{f\left(v_{\varepsilon}\left(y+y_{\varepsilon}\right)\right)}{v_{\varepsilon}\left(y+y_{\varepsilon}\right)} w_{\varepsilon}\left(y+y_{\varepsilon}\right)^{2} d y \rightarrow \infty .
\end{aligned}
$$

This is a contradiction to (2.16).

Step 2 : Case 1 cannot take place under (f5) with $a<\infty$.

As in Step 1, we extract a subsequence and we assume that (2.11), (2.12), (2.13) hold with $\chi\left(x_{0}\right) \neq 0$ and $w_{0}(y) \geq 0(\not \equiv 0)$. We shall prove that $w_{0}(y)$ satisfies

$$
-\Delta w_{0}+V\left(x_{0}\right) w_{0}=\left(\chi\left(x_{0}\right) a+\left(1-\chi\left(x_{0}\right)\right) \nu\right) w_{0} \quad \text { in } \mathbf{R}^{N} .
$$

Since the operator $-\Delta$ has no eigenvalues in $H^{1}\left(\mathbf{R}^{N}\right)$, this gives us the desired contradiction. To prove $(2.17)$, it suffices to show that

$$
\begin{aligned}
& \int_{\mathbf{R}^{N}} \nabla w_{\varepsilon}\left(y+y_{\varepsilon}\right) \nabla \varphi(y)+V\left(\varepsilon y+\varepsilon y_{\varepsilon}\right) w_{\varepsilon} \varphi d y \rightarrow \int_{\mathbf{R}^{N}} \nabla w_{0} \nabla \varphi+V\left(x_{0}\right) w_{0} \varphi d y \\
& \int_{\mathbf{R}^{N}} \frac{g\left(\varepsilon y+\varepsilon y_{\varepsilon}, v_{\varepsilon}\left(y+y_{\varepsilon}\right)\right)}{v_{\varepsilon}\left(y+y_{\varepsilon}\right)} w_{\varepsilon} \varphi d y \rightarrow\left(\chi\left(x_{0}\right) a+\left(1-\chi\left(x_{0}\right)\right) \nu\right) \int_{\mathbf{R}^{N}} w_{0} \varphi d y
\end{aligned}
$$


for any $\varphi \in C_{0}^{\infty}\left(\mathbf{R}^{N}\right)$. (2.18) is a direct consequence of (2.13). To prove (2.19) we take $R>1$ such that $\operatorname{supp} \varphi \subset B_{R}(0)$. Then $w_{\varepsilon} \rightarrow w_{0}$ strongly in $L^{2}\left(B_{R}(0)\right)$ and thus after extracting a sequence there exists $h(y) \in L^{2}\left(B_{R}(0)\right)$ such that $\left|w_{\varepsilon}(y)\right| \leq h(y)$ a.e. in $B_{R}(0)$ (see $[\mathbf{B r}]$, Theorem IV 9). Next since $a<\infty$, we can find a $C>0$ such that $|g(x, \xi) / \xi| \leq C$ for all $\xi>0$. Thus

$$
\left|\frac{g\left(\varepsilon y+\varepsilon y_{\varepsilon}, v_{\varepsilon}\left(y+y_{\varepsilon}\right)\right)}{v_{\varepsilon}\left(y+y_{\varepsilon}\right)} w_{\varepsilon} \varphi\right| \leq C\|\varphi\|_{\infty}\left|w_{\varepsilon}(y)\right| \leq C\|\varphi\|_{\infty} h(y) \in L^{1}\left(B_{R}(0)\right) .
$$

We have also

$$
\frac{g\left(\varepsilon y+\varepsilon y_{\varepsilon}, v_{\varepsilon}\left(y+y_{\varepsilon}\right)\right)}{v_{\varepsilon}\left(y+y_{\varepsilon}\right)} w_{\varepsilon} \rightarrow\left(\chi\left(x_{0}\right) a+\left(1-\chi\left(x_{0}\right)\right) \nu\right) w_{0}(y) \quad \text { a.e. in } B_{R}(0) .
$$

In fact, if $w_{0}(y)=0,(2.21)$ clearly holds. If $w_{0}(y) \neq 0$, we have $v_{\varepsilon}\left(y+y_{\varepsilon}\right) \rightarrow \infty$ and $(2.21)$ holds again. Combining (2.20) and (2.21), we get (2.19) by Lebesgue's theorem.

Finally we show Case 2 cannot take place.

Step 3 : Case 2 cannot take place.

Again we argue indirectly and assume Case 2 takes place. First we claim that $\left\|\chi_{\varepsilon} w_{\varepsilon}\right\|_{s+1} \rightarrow$ 0 . In fact $\left(\chi_{\varepsilon} w_{\varepsilon}\right)$ is a bounded sequence in $H^{1}\left(\mathbf{R}^{N}\right)$ by (2.8). Thus the following lemma implies $\left\|\chi_{\varepsilon} w_{\varepsilon}\right\|_{s+1} \rightarrow 0$.

Lemma 2.3. (c.f. $[\mathbf{L}])$ Suppose $\left(u_{j}\right) \subset H^{1}\left(\mathbf{R}^{N}\right)$ satisfies for some constant $C>0$

$$
\left\|u_{j}\right\|_{H^{1}\left(\mathbf{R}^{N}\right)} \leq C \quad \text { for all } j
$$

and

$$
\sup _{z \in \mathbf{R}^{N}} \int_{B_{1}(z)}\left|u_{j}\right|^{2} d y \rightarrow 0 .
$$

Then $\left\|u_{j}\right\|_{r} \rightarrow 0$ for $r \in\left(2, \frac{2 N}{N-2}\right)$ when $N \geq 3$ and $r \in(2, \infty)$ when $N=1,2$.

Following $[\mathbf{J}]$, for $L>1$ we compute

$$
J_{\varepsilon}\left(\frac{L}{\left\|v_{\varepsilon}\right\|_{H_{\varepsilon}}} v_{\varepsilon}\right)=\frac{1}{2} L^{2}-\int_{\mathbf{R}^{N}} \chi_{\varepsilon} F\left(L w_{\varepsilon}\right) d y-\int_{\mathbf{R}^{N}}\left(1-\chi_{\varepsilon}\right) \underline{F}\left(L w_{\varepsilon}\right) d y .
$$

By Lemma 1.2 (ii), we have $\int_{\mathbf{R}^{N}}\left(1-\chi_{\varepsilon}\right) \underline{F}\left(L w_{\varepsilon}\right) d y \leq \int_{\mathbf{R}^{N}} \frac{\nu L^{2}}{2}\left|w_{\varepsilon}\right|^{2} d y \leq \frac{1}{4} L^{2}$. Thus

$$
J_{\varepsilon}\left(\frac{L}{\left\|v_{\varepsilon}\right\|_{H_{\varepsilon}}} v_{\varepsilon}\right) \geq \frac{1}{4} L^{2}-\int_{\mathbf{R}^{N}} \chi_{\varepsilon} F\left(L w_{\varepsilon}\right) d y
$$


On the other hand, by (1.2) and our previous claim we have

$$
\begin{aligned}
\int_{\mathbf{R}^{N}} \chi_{\varepsilon} F\left(L w_{\varepsilon}\right) d y & \leq \delta L^{2}\left\|w_{\varepsilon}\right\|_{2}^{2}+C_{\delta} L^{s+1}\left\|\chi_{\varepsilon} w_{\varepsilon}\right\|_{s+1}\left\|w_{\varepsilon}\right\|_{s+1}^{s} \\
& \leq \frac{\delta L^{2}}{V_{0}^{2}}\left\|w_{\varepsilon}\right\|_{H_{\varepsilon}}^{2}+o(1) .
\end{aligned}
$$

Remarking that $\delta>0$ is arbitrary in (2.23), we obtain, combining (2.22) and (2.23),

$$
\liminf _{\varepsilon \rightarrow 0} J_{\varepsilon}\left(\frac{L}{\left\|v_{\varepsilon}\right\|_{H_{\varepsilon}}} v_{\varepsilon}\right) \geq \frac{1}{4} L^{2}
$$

Since $\left\|v_{\varepsilon}\right\|_{H_{\varepsilon}} \rightarrow \infty, \frac{L}{\left\|v_{\varepsilon}\right\|_{H_{\varepsilon}}} \in(0,1)$ for sufficiently small $\varepsilon>0$ and it follows that

$$
\max _{t \in[0,1]} J_{\varepsilon}\left(t v_{\varepsilon}\right) \geq J_{\varepsilon}\left(\frac{L}{\left\|v_{\varepsilon}\right\|_{H_{\varepsilon}}} v_{\varepsilon}\right) \geq \frac{1}{4} L^{2} .
$$

We recall that $J_{\varepsilon}\left(v_{\varepsilon}\right) \leq m_{2}$ independent of $\varepsilon$ and we choose $L>0$ so large that $m_{2}<\frac{1}{4} L^{2}$. Thus there exists $t_{\varepsilon} \in(0,1)$ such that

$$
J_{\varepsilon}\left(t_{\varepsilon} v_{\varepsilon}\right)=\max _{t \in[0,1]} J_{\varepsilon}\left(t v_{\varepsilon}\right)
$$

and since $L$ is arbitrary,

$$
J_{\varepsilon}\left(t_{\varepsilon} v_{\varepsilon}\right) \rightarrow \infty \quad \text { as } \varepsilon \rightarrow 0
$$

Now since $J_{\varepsilon}^{\prime}\left(t_{\varepsilon} v_{\varepsilon}\right)\left(t_{\varepsilon} v_{\varepsilon}\right)=0$ we can write

$$
\begin{aligned}
J_{\varepsilon}\left(t_{\varepsilon} v_{\varepsilon}\right) & =J_{\varepsilon}\left(t_{\varepsilon} v_{\varepsilon}\right)-\frac{1}{2} J_{\varepsilon}^{\prime}\left(t_{\varepsilon} v_{\varepsilon}\right)\left(t_{\varepsilon} v_{\varepsilon}\right)=\int_{\mathbf{R}^{N}} \widehat{G}\left(\varepsilon y, t_{\varepsilon} v_{\varepsilon}\right) d y \\
& \leq D^{k_{\nu}} \int_{\mathbf{R}^{N}} \widehat{G}\left(\varepsilon y, v_{\varepsilon}\right) d y=D^{k_{\nu}}\left(J_{\varepsilon}\left(v_{\varepsilon}\right)-\frac{1}{2} J_{\varepsilon}^{\prime}\left(v_{\varepsilon}\right) v_{\varepsilon}\right) \\
& \leq D^{k_{\nu}} m_{2}+o(1) .
\end{aligned}
$$

Here we use Corollary 1.3 (iv) and (2.5). Since (2.24) and (2.25) are incompatible, Case 2 cannot occur.

\section{Step 4 : Conclusion}

By Steps $1-3$, both Cases 1 and 2 cannot take place. Thus $\left\|v_{\varepsilon}\right\|_{H_{\varepsilon}}$ must stay bounded as $\varepsilon \rightarrow 0$.

Proof of Proposition 2.1. The proof of (i) in Proposition 2.1 can be done essentially in a similar way. However, since we fix $\varepsilon>0$ in Proposition 2.1, we need to modify the following points in Steps 1, 2 . 
In Step 1 for a given sequence $\left(v_{j}\right)$ we find $\left(y_{j}\right) \subset \mathbf{R}^{N}$ such that

$$
\int_{B_{1}\left(y_{j}\right)}\left|\chi_{\varepsilon} w_{j}\right|^{2} d y \rightarrow d>0
$$

Such $\left(y_{j}\right)$ satisfies $\varepsilon y_{j} \in N_{\varepsilon}(\Lambda)$ and we may assume $\varepsilon y_{j} \rightarrow x_{0} \in \overline{N_{\varepsilon}(\Lambda)}$, where $x_{0}$ satisfies $\chi\left(\varepsilon y+x_{0}\right) \not \equiv 0$ in $B_{1}(0)$.

In Step 2, instead of (2.17) we get

$$
-\Delta w_{0}+V\left(\varepsilon y+x_{0}\right) w_{0}=\left(\chi\left(\varepsilon y+x_{0}\right) a+\left(1-\chi\left(\varepsilon y+x_{0}\right)\right) \nu\right) w_{0} \quad \text { in } \mathbf{R}^{N}
$$

with $w_{0} \in H^{1}\left(\mathbf{R}^{N}\right)$ and $w_{0} \geq 0(\not \equiv 0)$. We remark that the maximum principle implies $w_{0}(y)>0$ for all $y \in \mathbf{R}^{N}$. Setting $\tilde{w}(x)=w_{0}\left(\left(x-x_{0}\right) / \varepsilon\right),(2.26)$ gives

$$
-\varepsilon^{2} \Delta \tilde{w}+V(x) \tilde{w}=(\chi(x) a+(1-\chi(x)) \nu) \tilde{w}
$$

We claim that this is impossible for sufficiently small $\varepsilon>0$. To show this we use an argument given to us by Stuart $[\mathbf{S t u}]$ and already used in $[\mathbf{J T 1}]$. We take $\ell>0$ small such that $\chi(x)=1$ and $V(x)<a$ in $B_{\ell}(0)$. Let $\lambda_{1}>0$ be the first eigenvalue of $-\Delta$ in $B_{\ell}(0)$ under Dirichlet boundary condition and let $\varphi_{1}(y)>0$ be a corresponding positive eigenfunction. Then multiplying $(2.27)$ by $\varphi_{1}(y)$, we obtain

$$
\int_{B_{\ell}(0)}-\varepsilon^{2} \Delta \tilde{w} \varphi_{1}+(V(x)-a) \tilde{w} \varphi_{1} d y=0 .
$$

Since

$$
\begin{aligned}
\int_{B_{\ell}(0)}-\Delta \tilde{w} \varphi_{1} d y & =\int_{B_{\ell}(0)}-\Delta \varphi_{1} \tilde{w} d y+\int_{\partial B_{\ell}(0)} \tilde{w} \frac{\partial \varphi_{1}}{\partial n} d S \\
& \leq \int_{B_{\ell}(0)} \lambda_{1} \tilde{w} \varphi_{1} d y
\end{aligned}
$$

where $n$ is the outer unit normal on $\partial B_{\ell}(0)$. We finally get

$$
\int_{B_{\ell}(0)}\left(V(x)-a+\varepsilon^{2} \lambda_{1}\right) \tilde{w} \varphi_{1} d y \geq 0 .
$$

But this is impossible since $V(x)-a+\varepsilon^{2} \lambda_{1}<0$ in $B_{\ell}(0)$ for small $\varepsilon>0$ and $\tilde{w} \varphi_{1}>0$ in $B_{\ell}(0)$.

To prove (ii) of Proposition 2.1 we fix $\varepsilon \in\left(0, \varepsilon_{1}\right]$ and $\left(v_{j}\right)$ satisfying $(2.2)-(2.3)$. By (i) $\left(v_{j}\right)$ is bounded in $H_{\varepsilon}$. After extracting a subsequence if necessary, we may assume that $v_{j} \rightarrow v_{0}$ weakly in $H_{\varepsilon}$. To show that this convergence is actually strong we follow 
del Pino-Felmer [DF1] who observe that it suffices to show that for any given $\delta>0$ there exists $R>0$ such that

$$
\limsup _{j \rightarrow \infty} \int_{|y| \geq R}\left|\nabla v_{j}\right|^{2}+V(\varepsilon y) v_{j}^{2} d y<\delta .
$$

Let $\eta_{R} \in C^{\infty}\left(\mathbf{R}^{N}, \mathbf{R}\right)$ be a cut-off function such that

$$
\begin{aligned}
& \eta_{R}(y)=0 \text { for }|y| \leq R / 2, \\
& \eta_{R}(y)=1 \text { for }|y| \geq R, \\
& \eta_{R}(y) \in[0,1] \text { for all } y \in \mathbf{R}^{N}, \\
& \left|\nabla \eta_{R}(y)\right| \leq C / R \text { for all } y \in \mathbf{R}^{N}
\end{aligned}
$$

for a suitable constant $C>0$.

Since $J_{\varepsilon}^{\prime}\left(v_{j}\right)\left(\eta_{R} v_{j}\right)=o(1)$, we have for sufficiently large $R>0$

$$
\begin{aligned}
\int_{\mathbf{R}^{N}}\left(\left|\nabla v_{j}\right|^{2}+V(\varepsilon y) v_{j}^{2}\right) \eta_{R}+v_{j} \nabla v_{j} \nabla \eta_{R} d y & =\int_{\mathbf{R}^{N}} \frac{f}{}\left(v_{j}\right) v_{j} \eta_{R} d y+o(1) \\
& \leq \nu \int_{\mathbf{R}^{N}}\left|v_{j}\right|^{2} \eta_{R} d y+o(1)
\end{aligned}
$$

Therefore

$$
\frac{1}{2} \int_{|y| \geq R}\left|\nabla v_{j}\right|^{2}+V(\varepsilon y) v_{j}^{2} d y \leq \frac{C}{R}\left\|v_{j}\right\|_{2}\left\|\nabla v_{j}\right\|_{2}+o(1)
$$

and (2.28) clearly follows.

From Propositions 2.1 and 2.2, we directly obtain

Corollary 2.4. There exists $\varepsilon_{1} \in\left(0, \varepsilon_{0}\right]$ such that for any $\varepsilon \in\left(0, \varepsilon_{1}\right]$ there exists a critical point $v_{\varepsilon} \in H_{\varepsilon}$ of $J_{\varepsilon}(v)$ satisfying

$$
J_{\varepsilon}\left(v_{\varepsilon}\right)=b_{\varepsilon}
$$

where $b_{\varepsilon} \in\left[m_{1}, m_{2}\right]$ is defined in (1.21)-(1.22). Moreover there exists a constant $M>0$ independent of $\varepsilon \in\left(0, \varepsilon_{1}\right]$ such that

$$
\left\|v_{\varepsilon}\right\|_{H_{\varepsilon}} \leq M \quad \text { for all } \varepsilon \in\left(0, \varepsilon_{1}\right]
$$

In the next section we will study the behavior of $v_{\varepsilon}$ as $\varepsilon \rightarrow 0$. 


\section{Concentration-compactness type argument for $J_{\varepsilon}(v)$}

In this section we study the behavior as $\varepsilon \rightarrow 0$ of the critical points $\left(v_{\varepsilon}\right)$ obtained in Corollary 2.4. More generally we study the behavior of functions $\left(v_{\varepsilon}\right)$ satisfying

$$
\begin{aligned}
& v_{\varepsilon} \in H_{\varepsilon}, \\
& J_{\varepsilon}\left(v_{\varepsilon}\right) \rightarrow c \in \mathbf{R}, \\
& \left(1+\left\|v_{\varepsilon}\right\|_{H_{\varepsilon}}\right)\left\|J_{\varepsilon}^{\prime}\left(v_{\varepsilon}\right)\right\|_{H_{\varepsilon}^{*}} \rightarrow 0, \\
& \left\|v_{\varepsilon}\right\|_{H_{\varepsilon}} \leq m,
\end{aligned}
$$

where the constants $c, m$ are independent of $\varepsilon$. We give a concentration-compactness type result depending on a parameter. For a standard setting of concentration-compactness principle, we refer to Lions $[\mathbf{L}]$ and Struwe $[\mathbf{S t r}]$.

To state our result, we need some definitions. For $x_{0} \in \mathbf{R}^{N}$, let $\Phi_{x_{0}}: H^{1}\left(\mathbf{R}^{N}\right) \rightarrow \mathbf{R}$ be given by

$$
\Phi_{x_{0}}(v)=\frac{1}{2} \int_{\mathbf{R}^{N}}|\nabla v|^{2}+V\left(x_{0}\right) v^{2} d y-\int_{\mathbf{R}^{N}} G\left(x_{0}, v\right) d y .
$$

For $x_{0} \in \mathbf{R}^{N}$ and $u, v \in H^{1}\left(\mathbf{R}^{N}\right)$ we write

$$
\begin{aligned}
& \langle u, v\rangle_{H_{\varepsilon}}=\int_{\mathbf{R}^{N}} \nabla u \nabla v+V(\varepsilon y) u v d y, \\
& \langle u, v\rangle_{x_{0}}=\int_{\mathbf{R}^{N}} \nabla u \nabla v+V\left(x_{0}\right) u v d y, \\
& |v|_{x_{0}}^{2}=\int_{\mathbf{R}^{N}}|\nabla v|^{2}+V\left(x_{0}\right) v^{2} d y .
\end{aligned}
$$

We choose a function $\psi(y) \in C_{0}^{\infty}\left(\mathbf{R}^{N}, \mathbf{R}\right)$ such that

$$
\begin{aligned}
& \psi(y)=1 \quad \text { for } y \in \Lambda, \\
& \psi(y) \in[0,1] \text { for all } y \in \mathbf{R}^{N} .
\end{aligned}
$$

We also define $\psi_{\varepsilon}(y)=\psi(\varepsilon y)$. Finally we set

$$
H(x, \xi)=-\frac{1}{2} V(x) \xi^{2}+\chi(x) F(\xi)+(1-\chi(x)) \underline{F}(\xi)
$$

and

$$
\Omega=\left\{x \in \mathbf{R}^{N} ; \sup _{\xi>0} H(x, \xi)>0\right\} .
$$

Remark 3.1. (i) $\Omega \subset \Lambda$ and $0 \in\left\{x \in \Lambda^{\prime} ; V(x)=\inf _{x \in \Lambda} V(x)\right\} \subset \Omega$.

(ii) If (f3) or (f5) with $a=\infty$ holds, $\Omega=\Lambda$.

Now we can state the main result of this section. 
Proposition 3.2. Assume that $f(\xi)$ satisfies (f0)-(f2) and that $\left(v_{\varepsilon}\right)_{\varepsilon \in\left(0, \varepsilon_{1}\right]}$ satisfies (3.1)(3.4). Then there exists a subsequence $\varepsilon_{j} \rightarrow 0, \ell \in \mathbf{N} \cup\{0\}$, sequences $\left(y_{\varepsilon_{j}}^{k}\right) \subset \mathbf{R}^{N}, x^{k} \in \Omega$, $\omega^{k} \in H^{1}\left(\mathbf{R}^{N}\right) \backslash\{0\}(k=1,2, \cdots, \ell)$ such that

(i) $\left|y_{\varepsilon_{j}}^{k}-y_{\varepsilon_{j}}^{k^{\prime}}\right| \rightarrow \infty$ as $j \rightarrow \infty$ for $k \neq k^{\prime}$.

(ii) $\varepsilon_{j} y_{\varepsilon_{j}}^{k} \rightarrow x^{k} \in \Omega$ as $j \rightarrow \infty$.

(iii) $\omega^{k} \not \equiv 0$ and $\Phi_{x^{k}}^{\prime}\left(\omega^{k}\right)=0$.

(iv) $\left\|v_{\varepsilon_{j}}-\psi_{\varepsilon_{j}}\left(\sum_{k=1}^{\ell} \omega^{k}\left(y-y_{\varepsilon_{j}}^{k}\right)\right)\right\|_{H_{\varepsilon_{j}}} \rightarrow 0$ as $j \rightarrow \infty$.

(v) $J_{\varepsilon_{j}}\left(v_{\varepsilon_{j}}\right) \rightarrow \sum_{k=1}^{\ell} \Phi_{x^{k}}\left(\omega^{k}\right)$.

Remark 3.3. (i) When $\ell=0$ in the statement of Proposition 3.2, it means that

$$
\left\|v_{\varepsilon_{j}}\right\|_{H_{\varepsilon_{j}}} \rightarrow 0 \quad \text { and } \quad J_{\varepsilon_{j}}\left(v_{\varepsilon_{j}}\right) \rightarrow 0
$$

(ii) A closely related result to Proposition 3.2 is obtained by Gui [Gu, Proposition 2.2]. We remark that in [Gu, Proposition 2.2] it is assumed, besides other conditions,

$$
0 \leq f_{\xi}(\xi) \leq a_{1}+a_{2} \xi^{p-1}
$$

for some $a_{1}, a_{2}>0$ and $p \in\left(1, \frac{N+2}{N-2}\right)$, so Proposition 3.2 may be regarded as a generalization.

Before proving Proposition 3.2, we remark that the functional $\Phi_{x_{0}}(v)$ corresponds to the limit problem :

$$
-\Delta v+V\left(x_{0}\right) v=g\left(x_{0}, v\right) \text { in } \mathbf{R}^{N} .
$$

A typical feature of the limit problem is its $x$-independence, that is, (3.5) is an autonomous equation. The following lemma is important in the proof of Proposition 3.2.

Lemma 3.4. Assume that $f(\xi)$ satisfies (f0)-(f2). Then

(i) $\Phi_{x_{0}}(v)$ has non-zero critical points if and only if $x_{0} \in \Omega$.

(ii) There exists a constant $\delta_{1}>0$ independent of $x_{0} \in \mathbf{R}^{N}$ such that

$$
|v|_{x_{0}} \geq \delta_{1}
$$

for any non-zero critical point $v(y)$ of $\Phi_{x_{0}}(v)$.

Although the proof of Lemma 3.4 is not complicated, we postpone it to Section 4 . 
Remark 3.5. Since we don't assume any growth condition on $V(x)$, in general $\omega \notin H_{\varepsilon}$ for a critical point $\omega(y)$ of $\Phi_{x_{0}}(v)$ and $\varepsilon>0$. This motivates the introduction of a cut-off function $\psi_{\varepsilon}(y)$ in (iv) of Proposition 3.2. We remark that $\sup \psi(\varepsilon y) V(\varepsilon y)<\infty$ and $\psi_{\varepsilon}$ has the following properties :

(i) For any $w \in H^{1}\left(\mathbf{R}^{N}\right), \psi_{\varepsilon} w \in H_{\varepsilon}$ and there is a constant $C>0$ independent of $\varepsilon$ such that

$$
\left\|\psi_{\varepsilon} w\right\|_{H_{\varepsilon}} \leq C\|w\|_{H^{1}\left(\mathbf{R}^{N}\right)} \text { for all } w \in H^{1}\left(\mathbf{R}^{N}\right) .
$$

(ii) For any $w \in H^{1}\left(\mathbf{R}^{N}\right)$ and for any sequence $\left(z_{\varepsilon}\right) \subset \mathbf{R}^{N}$ satisfying $\varepsilon z_{\varepsilon} \rightarrow x_{0}$ for some $x_{0} \in \Lambda$ we have

$$
\begin{aligned}
& \left\|\psi_{\varepsilon}(y) w\left(y-z_{\varepsilon}\right)\right\|_{H_{\varepsilon}}^{2} \\
& =\int_{\mathbf{R}^{N}}\left|\nabla\left(\psi_{\varepsilon}(y) w\left(y-z_{\varepsilon}\right)\right)\right|^{2}+V(\varepsilon y) \psi_{\varepsilon}(y)^{2} w\left(y-z_{\varepsilon}\right)^{2} d y \\
& =\int_{\mathbf{R}^{N}}\left|\nabla\left(\psi\left(\varepsilon y+\varepsilon z_{\varepsilon}\right) w(y)\right)\right|^{2}+V\left(\varepsilon y+\varepsilon z_{\varepsilon}\right) \psi\left(\varepsilon y+\varepsilon z_{\varepsilon}\right)^{2} w(y)^{2} d y \\
& \rightarrow \int_{\mathbf{R}^{N}}|\nabla w|^{2}+V\left(x_{0}\right) w^{2} d y \\
& =|w|_{x_{0}}^{2} \text { as } \varepsilon \rightarrow 0 .
\end{aligned}
$$

Proof of Proposition 3.2. The proof of Proposition 3.2 consists of several steps. For simplicity of notation, we write $\varepsilon$ instead of $\varepsilon_{j}$. We take subsequences repeatedly and we also write just $\varepsilon$.

Step 1 : Extracting a subsequence if necessary, we can assume that $v_{\varepsilon} \rightarrow v_{0}$ weakly in $H^{1}\left(\mathbf{R}^{N}\right)$ with $v_{0}(y)$ a critical point of $\Phi_{0}(v)$.

First we remark that $\left\|v_{\varepsilon}\right\|_{H^{1}\left(\mathbf{R}^{N}\right)} \leq m$ follows from (3.4) and (1.19). Thus $\left(v_{\varepsilon}\right)$ is bounded in $H^{1}\left(\mathbf{R}^{N}\right)$ and we may assume that

$$
v_{\varepsilon} \rightarrow v_{0} \in H^{1}\left(\mathbf{R}^{N}\right) \quad \text { weakly in } H^{1}\left(\mathbf{R}^{N}\right)
$$

In a standard way we can see that $v_{0}(y)$ is a critical point of $\Phi_{0}(v)$. Indeed for any $\varphi(y) \in C_{0}^{\infty}\left(\mathbf{R}^{N}\right)$, we have $J_{\varepsilon}^{\prime}\left(v_{\varepsilon}\right) \varphi \rightarrow 0$, i.e.,

$$
\int_{\mathbf{R}^{N}} \nabla v_{\varepsilon} \nabla \varphi+V(\varepsilon y) v_{\varepsilon} \varphi-g\left(\varepsilon y, v_{\varepsilon}\right) \varphi d y \rightarrow 0 .
$$

Thus we have

$$
\int_{\mathbf{R}^{N}} \nabla v_{0} \nabla \varphi+V(0) v_{0} \varphi-g\left(0, v_{0}\right) \varphi d y=0 \quad \text { for all } \varphi \in C_{0}^{\infty}\left(\mathbf{R}^{N}\right) .
$$


That is, $\Phi_{0}^{\prime}\left(v_{0}\right) \varphi=0$ for all $\varphi \in C_{0}^{\infty}\left(\mathbf{R}^{N}\right)$. Since $C_{0}^{\infty}\left(\mathbf{R}^{N}\right)$ is dense in $H^{1}\left(\mathbf{R}^{N}\right)$, we have $\Phi_{0}^{\prime}\left(v_{0}\right)=0$.

If $v_{0}(y) \not \equiv 0$, we set $y_{\varepsilon}^{1}=0$ and $\omega^{1}=v_{0}$.

Step 2: Suppose there exist $n \in \mathbf{N} \cup\{0\},\left(y_{\varepsilon}^{k}\right) \subset \mathbf{R}^{N}, x^{k} \in \Omega, \omega^{k} \in H^{1}\left(\mathbf{R}^{N}\right)(k=$ $1,2, \cdots, n$ ) such that (CC.1), (CC.2), (CC.3) of Proposition 3.2 hold for $k=1,2, \cdots, n$ and

$$
v_{\varepsilon}\left(y+y_{\varepsilon}^{k}\right) \rightarrow \omega^{k}(y) \quad \text { weakly in } H^{1}\left(\mathbf{R}^{N}\right) \text { for } k=1,2, \cdots, n .
$$

Assume moreover that

$$
\sup _{y \in \mathbf{R}^{N}} \int_{B_{1}(y)}\left|v_{\varepsilon}-\psi_{\varepsilon} \sum_{k=1}^{n} \omega^{k}\left(y-y_{\varepsilon}^{k}\right)\right|^{2} d y \rightarrow 0 .
$$

Then

$$
\left\|v_{\varepsilon}-\psi_{\varepsilon} \sum_{k=1}^{n} \omega^{k}\left(y-y_{\varepsilon}^{k}\right)\right\|_{H_{\varepsilon}} \rightarrow 0 .
$$

We set $\zeta_{\varepsilon}(y)=v_{\varepsilon}(y)-\psi_{\varepsilon}(y) \sum_{k=1}^{n} \omega^{k}\left(y-y_{\varepsilon}^{k}\right)$. Using (3.6), we have

$$
\left\|\zeta_{\varepsilon}\right\|_{H^{1}\left(\mathbf{R}^{N}\right)} \leq\left\|\zeta_{\varepsilon}\right\|_{H_{\varepsilon}} \leq\left\|v_{\varepsilon}\right\|_{H_{\varepsilon}}+\left\|\psi_{\varepsilon} \sum_{k=1}^{n} \omega^{k}\left(y-y_{\varepsilon}^{k}\right)\right\|_{H_{\varepsilon}} \leq m+C \sum_{k=1}^{n}\left\|\omega^{k}\right\|_{H^{1}\left(\mathbf{R}^{N}\right)} .
$$

Thus $\zeta_{\varepsilon}$ is bounded in $H^{1}\left(\mathbf{R}^{N}\right)$. It follows from (3.8) and Lemma 2.3 that $\left\|\zeta_{\varepsilon}\right\|_{s+1} \rightarrow 0$ as $\varepsilon \rightarrow 0$. Now we compute

$$
\begin{aligned}
\left\|\zeta_{\varepsilon}\right\|_{H_{\varepsilon}}^{2} & =\left\langle v_{\varepsilon}-\psi_{\varepsilon} \sum_{k=1}^{n} \omega^{k}\left(y-y_{\varepsilon}^{k}\right), \zeta_{\varepsilon}\right\rangle_{H_{\varepsilon}} \\
& =\left\langle v_{\varepsilon}, \zeta_{\varepsilon}\right\rangle_{H_{\varepsilon}}-\sum_{k=1}^{n}\left\langle\psi_{\varepsilon} \omega^{k}\left(y-y_{\varepsilon}^{k}\right), \zeta_{\varepsilon}\right\rangle_{H_{\varepsilon}}
\end{aligned}
$$

We claim that for all $k=1, \cdots, n$

$$
\left\langle\psi_{\varepsilon} \omega^{k}\left(y-y_{\varepsilon}^{k}\right), \zeta_{\varepsilon}\right\rangle_{H_{\varepsilon}}=\left\langle\omega^{k}\left(y-y_{\varepsilon}^{k}\right), \psi_{\varepsilon} \zeta_{\varepsilon}\right\rangle_{x^{k}}+o(1) .
$$

In fact,

$$
\begin{aligned}
& \left\langle\psi_{\varepsilon} \omega^{k}\left(y-y_{\varepsilon}^{k}\right), \zeta_{\varepsilon}\right\rangle_{H_{\varepsilon}}-\left\langle\omega^{k}\left(y-y_{\varepsilon}^{k}\right), \psi_{\varepsilon} \zeta_{\varepsilon}\right\rangle_{x^{k}} \\
= & \int_{\mathbf{R}^{N}} \nabla\left(\psi_{\varepsilon} \omega^{k}\left(y-y_{\varepsilon}^{k}\right)\right) \nabla \zeta_{\varepsilon}-\nabla\left(\omega^{k}\left(y-y_{\varepsilon}^{k}\right)\right) \nabla\left(\psi_{\varepsilon} \zeta_{\varepsilon}\right) d y
\end{aligned}
$$




$$
\begin{aligned}
& +\int_{\mathbf{R}^{N}}\left(V(\varepsilon y)-V\left(x^{k}\right)\right) \psi_{\varepsilon} \omega^{k}\left(y-y_{\varepsilon}^{k}\right) \zeta_{\varepsilon}(y) d y \\
= & \int_{\mathbf{R}^{N}} \omega^{k}\left(y-y_{\varepsilon}^{k}\right) \nabla \psi_{\varepsilon} \nabla \zeta_{\varepsilon}-\nabla \psi_{\varepsilon} \nabla \omega^{k}\left(y-y_{\varepsilon}^{k}\right) \zeta_{\varepsilon} d y \\
& +\int_{\mathbf{R}^{N}}\left(V\left(\varepsilon y+\varepsilon y_{\varepsilon}^{k}\right)-V\left(x^{k}\right)\right) \psi\left(\varepsilon y+\varepsilon y_{\varepsilon}^{k}\right) \omega^{k}(y) \zeta_{\varepsilon}\left(y+y_{\varepsilon}^{k}\right) d y \\
= & (I)+(I I) .
\end{aligned}
$$

Since $\left\|\nabla \psi_{\varepsilon}\right\|_{\infty} \rightarrow 0$ as $\varepsilon \rightarrow 0$, we get $(I) \rightarrow 0$ from the boundedness of $\left\|\zeta_{\varepsilon}\right\|_{H^{1}\left(\mathbf{R}^{N}\right)}$. We remark that

$$
\left(V\left(\varepsilon y+\varepsilon y_{\varepsilon}^{k}\right)-V\left(x^{k}\right)\right) \psi\left(\varepsilon y+\varepsilon y_{\varepsilon}^{k}\right)
$$

is bounded in $L^{\infty}\left(\mathbf{R}^{N}\right)$. By (3.7) and (CC.1), we have

$$
\zeta_{\varepsilon}\left(y+y_{\varepsilon}^{k}\right) \rightarrow 0 \quad \text { weakly in } H^{1}\left(\mathbf{R}^{N}\right) \text { and strongly in } L_{l o c}^{2}\left(\mathbf{R}^{N}\right) .
$$

Thus $(I I) \rightarrow 0$ and we get (3.11).

Combining (3.10) and (3.11), we have

$$
\begin{aligned}
\left\|\zeta_{\varepsilon}\right\|_{H_{\varepsilon}}^{2}= & \left\langle v_{\varepsilon}, \zeta_{\varepsilon}\right\rangle_{H_{\varepsilon}}-\sum_{k=1}^{n}\left\langle\omega^{k}\left(y-y_{\varepsilon}^{k}\right), \psi_{\varepsilon} \zeta_{\varepsilon}\right\rangle_{x^{k}}+o(1) \\
= & J_{\varepsilon}^{\prime}\left(v_{\varepsilon}\right) \zeta_{\varepsilon}+\int_{\mathbf{R}^{N}} g\left(\varepsilon y, v_{\varepsilon}\right) \zeta_{\varepsilon} d y \\
& -\sum_{k=1}^{n}\left(\Phi_{x^{k}}^{\prime}\left(\omega^{k}\left(y-y_{\varepsilon}^{k}\right)\right)\left(\psi_{\varepsilon} \zeta_{\varepsilon}\right)+\int_{\mathbf{R}^{N}} g\left(x^{k}, \omega^{k}\left(y-y_{\varepsilon}^{k}\right)\right) \psi_{\varepsilon} \zeta_{\varepsilon} d y\right)+o(1) \\
= & \int_{\mathbf{R}^{N}} g\left(\varepsilon y, v_{\varepsilon}\right) \zeta_{\varepsilon} d y-\sum_{k=1}^{n} \int_{\mathbf{R}^{N}} g\left(x^{k}, \omega^{k}\left(y-y_{\varepsilon}^{k}\right)\right) \psi_{\varepsilon} \zeta_{\varepsilon} d y+o(1) \\
= & (I I I)-\sum_{k=1}^{n}(I V)+o(1)
\end{aligned}
$$

By Corollary 1.3 (iii),

$$
|(I I I)| \leq \delta\left\|v_{\varepsilon}\right\|_{2}\left\|\zeta_{\varepsilon}\right\|_{2}+C_{\delta}\left\|v_{\varepsilon}\right\|_{s+1}^{s}\left\|\zeta_{\varepsilon}\right\|_{s+1}
$$

Since $\left\|\zeta_{\varepsilon}\right\|_{s+1} \rightarrow 0$ and $\left\|v_{\varepsilon}\right\|_{2},\left\|\zeta_{\varepsilon}\right\|_{2}$ are bounded, we can see that $(I I I) \rightarrow 0$. For $(I V)$, we have

$$
(I V)=\int_{\mathbf{R}^{N}} g\left(x^{k}, \omega^{k}(y)\right) \psi\left(\varepsilon y+\varepsilon y_{\varepsilon}^{k}\right) \zeta_{\varepsilon}\left(y+y_{\varepsilon}^{k}\right) d y .
$$

Recalling (3.12), we get $(I V) \rightarrow 0$. Thus we have proved that $\left\|\zeta_{\varepsilon}\right\|_{H_{\varepsilon}} \rightarrow 0$. That is (3.9). 
Next we consider the case where the conclusion (3.9) does not hold. In this case we can find a sequence $z_{\varepsilon}$ satisfying (3.13) below.

Step 3 : Suppose there exist $n \in \mathbf{N} \cup\{0\},\left(y_{\varepsilon}^{k}\right) \subset \mathbf{R}^{N}, x^{k} \in \Omega, \omega^{k} \in H^{1}\left(\mathbf{R}^{N}\right) \backslash\{0\}$ $(k=1,2, \cdots, n)$ such that (CC.1), (CC.2), (CC.3), (3.7) hold. Assume moreover that there exists $z_{\varepsilon} \in \mathbf{R}^{N}$ such that

$$
\int_{B_{1}\left(z_{\varepsilon}\right)}\left|v_{\varepsilon}-\psi_{\varepsilon} \sum_{k=1}^{n} \omega^{k}\left(y-y_{\varepsilon}^{k}\right)\right|^{2} d y \rightarrow c>0
$$

for some $c>0$. Then there exists $x^{k+1} \in \Omega$ and $\omega^{k+1}(y) \in H^{1}\left(\mathbf{R}^{N}\right) \backslash\{0\}$ such that

$$
\begin{aligned}
& \left|z_{\varepsilon}-y_{\varepsilon}^{k}\right| \rightarrow \infty \quad \text { for all } k=1,2, \cdots, n, \\
& \varepsilon z_{\varepsilon} \rightarrow x^{k+1} \in \Omega, \\
& v_{\varepsilon}\left(y+z_{\varepsilon}\right) \rightarrow \omega^{k+1}(y) \not \equiv 0 \quad \text { weakly in } H^{1}\left(\mathbf{R}^{N}\right), \\
& \Phi_{x^{k+1}}^{\prime}\left(\omega^{k+1}\right)=0 .
\end{aligned}
$$

It is standard to check that $z_{\varepsilon}$ satisfies (3.14) and that there exists $\omega^{k+1} \in H^{1}\left(\mathbf{R}^{N}\right) \backslash\{0\}$ satisfying (3.16). Let us prove (3.15). First we show that $\lim _{\sup } \sup _{\varepsilon \rightarrow 0}\left|\varepsilon z_{\varepsilon}\right|<\infty$. We argue indirectly and assume $\left|\varepsilon z_{\varepsilon}\right| \rightarrow \infty$. For any $\varphi \in C_{0}^{\infty}\left(\mathbf{R}^{N}\right)$ with $\varphi \geq 0$, we have

$$
J_{\varepsilon}^{\prime}\left(v_{\varepsilon}\right)\left(\varphi\left(y-z_{\varepsilon}\right) v_{\varepsilon}\right) \rightarrow 0
$$

That is,

$$
\begin{gathered}
\int_{\mathbf{R}^{N}} \nabla v_{\varepsilon}\left(y+z_{\varepsilon}\right) \nabla\left(\varphi(y) v_{\varepsilon}\left(y+z_{\varepsilon}\right)\right)+V\left(\varepsilon y+\varepsilon z_{\varepsilon}\right) v_{\varepsilon}\left(y+z_{\varepsilon}\right)^{2} \varphi(y) d y \\
-\int_{\mathbf{R}^{N}} g\left(\varepsilon y+\varepsilon z_{\varepsilon}, v_{\varepsilon}\left(y+z_{\varepsilon}\right)\right) v_{\varepsilon}\left(y+z_{\varepsilon}\right) \varphi(y) d y \rightarrow 0 .
\end{gathered}
$$

We observe that $g\left(\varepsilon y+\varepsilon z_{\varepsilon}, v_{\varepsilon}\left(y+z_{\varepsilon}\right)\right)=\underline{f}\left(v_{\varepsilon}\left(y+z_{\varepsilon}\right)\right)$ on $\operatorname{supp} \varphi$ for small $\varepsilon$ under the assumption $\left|\varepsilon z_{\varepsilon}\right| \rightarrow \infty$. Also since

$$
\begin{aligned}
& \int_{\mathbf{R}^{N}} \nabla v_{\varepsilon}\left(y+z_{\varepsilon}\right)(\nabla \varphi)(y) v_{\varepsilon}\left(y+z_{\varepsilon}\right) d y \rightarrow \int_{\mathbf{R}^{N}} \nabla \omega^{k+1}(\nabla \varphi) \omega^{k+1} d y, \\
& \liminf _{\varepsilon \rightarrow 0} \int_{\mathbf{R}^{N}}\left|\nabla v_{\varepsilon}\left(y+z_{\varepsilon}\right)\right|^{2} \varphi d y \geq \int_{\mathbf{R}^{N}}\left|\nabla \omega^{k+1}\right|^{2} \varphi d y
\end{aligned}
$$

we deduce from (3.18) that

$$
\int_{\mathbf{R}^{N}}\left|\nabla \omega^{k+1}\right|^{2} \varphi+\nabla \omega^{k+1}(\nabla \varphi) \omega^{k+1}+V_{0}\left(\omega^{k+1}\right)^{2} \varphi-\underline{f}\left(\omega^{k+1}\right) \omega^{k+1} \varphi d y \leq 0 .
$$


We choose $\tilde{\varphi}(y) \in C_{0}^{\infty}\left(\mathbf{R}^{N}\right)$ satisfying $\tilde{\varphi}(y) \geq 0, \tilde{\varphi}(0)=1$ and set $\varphi(y)=\tilde{\varphi}(y / R)$ in $(3.19)$. Taking a limit as $R \rightarrow \infty$, in (3.19) we get

$$
\int_{\mathbf{R}^{N}}\left|\nabla \omega^{k+1}\right|^{2}+V_{0}\left(\omega^{k+1}\right)^{2}-\underline{f}\left(\omega^{k+1}\right) \omega^{k+1} d y \leq 0 .
$$

By Lemma 1.2 (i)-(ii), this implies that $\omega^{k+1} \equiv 0$, which contradicts with (3.16). Thus $\limsup _{\varepsilon \rightarrow 0}\left|\varepsilon z_{\varepsilon}\right|<\infty$ and we may assume that $\varepsilon z_{\varepsilon} \rightarrow x^{k+1} \in \mathbf{R}^{N}$. At this point we can get (3.17) in a standard way and since $\omega^{k+1}$ is a non-trivial critical point of $\Phi_{x^{k+1}}(v)$, we deduce that $x^{k+1} \in \Omega$ by Lemma 3.4 (i).

\section{Step 4 : Conclusion}

We follow a recursive procedure. If the weak limit $v_{0}(y)$ of $v_{\varepsilon}(y)$ provided by Step 1 is not 0 , we set $y_{\varepsilon}^{1}=0, x^{1}=0, \omega^{1}(y)=v_{0}(y)$. Then if $\left\|v_{\varepsilon}-\psi_{\varepsilon} \omega^{1}\right\|_{H_{\varepsilon}} \rightarrow 0$ (or $\left\|v_{\varepsilon}\right\|_{H_{\varepsilon}} \rightarrow 0$ in case $\left.v_{0} \equiv 0\right)$, we are done. Otherwise, by Step 2, we can see that (3.8) does not take place, and that there exists a sequence $\left(z_{\varepsilon}\right)$ satisfying the assumption (3.13) of Step 3. Applying Step 3, we can find $x^{2}$ and $\omega^{2}(y)$ (or $x^{1}$ and $\omega^{1}(y)$ in case $v_{0} \equiv 0$ ) satisfying (3.13)-(3.17). We set $y_{\varepsilon}^{2}=z_{\varepsilon}\left(\right.$ or $y_{\varepsilon}^{1}=z_{\varepsilon}$ in case $\left.v_{0} \equiv 0\right)$. If $\left\|z_{\varepsilon}-\psi_{\varepsilon}\left(\omega^{1}(y)+\omega^{2}\left(y-y_{\varepsilon}^{2}\right)\right)\right\|_{H_{\varepsilon}} \rightarrow 0$, we are done. Otherwise, we use Steps 2, 3 and we continue this procedure. Now we need to prove that it stops after a finite number of steps.

First we prove that under the assumptions (CC.1)-(CC.3) and (3.7)

$$
\lim _{\varepsilon \rightarrow 0}\left\|v_{\varepsilon}-\psi_{\varepsilon} \sum_{k=1}^{n} \omega^{k}\left(y-y_{\varepsilon}^{k}\right)\right\|_{H_{\varepsilon}}^{2}=\lim _{\varepsilon \rightarrow 0}\left\|v_{\varepsilon}\right\|_{H_{\varepsilon}}^{2}-\sum_{k=1}^{n} \mid \mathbf{|} \omega^{k} \mathbf{I}_{x^{k}}^{2}
$$

In fact,

$$
\begin{aligned}
\left\|v_{\varepsilon}-\psi_{\varepsilon} \sum_{k=1}^{n} \omega^{k}\left(y-y_{\varepsilon}^{k}\right)\right\|_{H_{\varepsilon}}^{2}= & \left\|v_{\varepsilon}\right\|_{H_{\varepsilon}}^{2}-2 \sum_{k=1}^{n}\left\langle v_{\varepsilon}, \psi_{\varepsilon} \omega^{k}\left(y-y_{\varepsilon}^{k}\right)\right\rangle_{H_{\varepsilon}} \\
& +\sum_{k, k^{\prime}}\left\langle\psi_{\varepsilon} \omega^{k}\left(y-y_{\varepsilon}^{k}\right), \psi_{\varepsilon} \omega^{k^{\prime}}\left(y-y_{\varepsilon}^{k^{\prime}}\right)\right\rangle_{H_{\varepsilon}}
\end{aligned}
$$

and we have

$$
\begin{aligned}
& \left\langle v_{\varepsilon}, \psi_{\varepsilon} \omega^{k}\left(y-y_{\varepsilon}^{k}\right)\right\rangle_{H_{\varepsilon}} \\
& =\int_{\mathbf{R}^{N}} \nabla v_{\varepsilon}\left(y+y_{\varepsilon}^{k}\right) \nabla\left(\psi\left(\varepsilon y+\varepsilon y_{\varepsilon}^{k}\right) \omega^{k}(y)\right)+V\left(\varepsilon y+\varepsilon y_{\varepsilon}^{k}\right) \psi\left(\varepsilon y+\varepsilon y_{\varepsilon}^{k}\right) v_{\varepsilon}\left(y+y_{\varepsilon}^{k}\right) \omega^{k}(y) d y \\
& \rightarrow \int_{\mathbf{R}^{N}}\left|\nabla \omega^{k}\right|^{2}+V\left(x^{k}\right)\left(\omega^{k}\right)^{2} d y \\
& =\left|\omega^{k}\right|_{x_{k}}^{2} .
\end{aligned}
$$


In a similar way, we have

$$
\left\langle\psi_{\varepsilon} \omega^{k}\left(y-y_{\varepsilon}^{k}\right), \psi_{\varepsilon} \omega^{k^{\prime}}\left(y-y_{\varepsilon}^{k^{\prime}}\right)\right\rangle_{H_{\varepsilon}} \rightarrow \begin{cases}0 & \text { if } k \neq k^{\prime}, \\ \left|\omega^{k}\right|_{x_{k}}^{2} & \text { if } k=k^{\prime} .\end{cases}
$$

Thus (3.20) follows from (3.21)-(3.23).

Now from (3.20) it follows that

$$
\sum_{k=1}^{n}\left|\omega^{k}\right|_{x^{k}}^{2} \leq \lim _{\varepsilon \rightarrow 0}\left\|v_{\varepsilon}\right\|_{H_{\varepsilon}}^{2}
$$

and using Lemma 3.4 (ii), (3.4), we deduce

$$
\delta_{1} n \leq \lim _{\varepsilon \rightarrow 0}\left\|v_{\varepsilon}\right\|_{H_{\varepsilon}}^{2} \leq m^{2} .
$$

Thus the procedure to find $\left(y_{\varepsilon}^{k}\right), x^{k}, \omega^{k}$ ends after a finite number of steps. Therefore we can find $\ell \in \mathbf{N} \cup\{0\},\left(y_{\varepsilon}^{k}\right), x^{k}, \omega^{k}(k=1,2, \cdots, \ell)$ such that (CC.1)-(CC.4) hold. (CC.5) follows in a standard way from (CC.1)-(CC.4). This ends the proof of Proposition 3.2. 


\section{The functionals $\Phi_{x_{0}}(v)$}

In this section we study the limit functionals $\Phi_{x_{0}}(v)$ for $x_{0} \in \mathbf{R}^{N}$. Since $\Phi_{x_{0}}(v)$ is autonomous, we can deal with $\Phi_{x_{0}}(v)$ in a space of radially symmetric functions and we have the following existence result. It is due to Berestycki-Lions [BL] for $N \geq 3$ and Berestycki-Gallouët-Kavian [BGK] for $N=2$.

Proposition 4.1. ([BL], $[\mathbf{B G K}])$. Assume that $h(\xi)$ satisfies

(h0) $h(\xi) \in C(\mathbf{R}, \mathbf{R})$ is continuous and odd.

(h1) $-\infty<\liminf _{\xi \rightarrow 0} \frac{h(\xi)}{\xi} \leq \lim \sup _{\xi \rightarrow 0} \frac{h(\xi)}{\xi}<0$ for $N \geq 3$, $\lim _{\xi \rightarrow 0} \frac{h(\xi)}{\xi} \in(-\infty, 0)$ for $N=2$.

(h2) When $N \geq 3, \lim _{\xi \rightarrow \infty} \frac{h(\xi)}{\xi^{\frac{2 N}{N-2}}}=0$, when $N=2$, for any $\alpha>0$ there exists $C_{\alpha}>0$ such that

$$
|h(\xi)| \leq C_{\alpha} e^{\alpha \xi^{2}} \quad \text { for all } \xi
$$

Then the problem

$$
-\Delta u=h(u) \quad \text { in } \mathbf{R}^{N}, \quad u(x) \in H^{1}\left(\mathbf{R}^{N}\right)
$$

has a non-zero solution if and only if the following condition is satisfied.

(h3) There exists $\xi_{0}>0$ such that $H\left(\xi_{0}\right)>0$, where $H(\xi)=\int_{0}^{\xi} h(\tau) d \tau$. Moreover under (h0)-(h3), (4.1) has a least energy solution $u(x)$ which satisfies $u(x)>0$ and is radially symmetric in $\mathbf{R}^{N}$.

Here by a least energy solution we mean a solution $\omega(x)$ which satisfies $\tilde{I}(\omega)=m$, where

$$
\begin{aligned}
& m=\inf \left\{\tilde{I}(u) ; u \in H^{1}\left(\mathbf{R}^{N}\right) \backslash\{0\} \text { is a solution of }(4.1)\right\} \\
& \tilde{I}(u)=\int_{\mathbf{R}^{N}} \frac{1}{2}|\nabla u|^{2}-H(u) d y .
\end{aligned}
$$

It is also shown that $m>0$.

In our recent work [JT2], we have revisited (4.1) and enlighten a mountain pass characterization of least energy solutions.

Proposition 4.2. ([JT2]). Assume that (h0)-(h3) hold. Then $\tilde{I}(u)$ has a mountain pass geometry and there holds that

$$
b=m,
$$


where $m$ is defined in (4.2) and $b$ is the mountain pass value for $\tilde{I}(u)$;

$$
\begin{aligned}
b & =\inf _{\gamma \in \Gamma} \max _{t \in[0,1]} \tilde{I}(\gamma(t)), \\
\Gamma & =\left\{\gamma(t) \in C\left([0,1], H^{1}\left(\mathbf{R}^{N}\right)\right) ; \gamma(0)=0, \tilde{I}(\gamma(1))<0\right\} .
\end{aligned}
$$

Moreover for any least energy solution $\omega(x)$ of (4.1) there exists a path $\gamma(t) \in \Gamma$ such that

$$
\begin{aligned}
& \tilde{I}(\gamma(t)) \leq m=\tilde{I}(\omega) \text { for all } t \in[0,1] \\
& \omega \in \gamma([0,1]) .
\end{aligned}
$$

We remark that the relation (4.3) is usually obtained under the assumption of the monotonicity of $(0, \infty) \rightarrow \mathbf{R} ; \xi \mapsto \frac{h(\xi)}{\xi}$. Under this assumption, $\gamma(t)=t L \omega$ gives a path satisfying (4.4)-(4.5) for large $L>1$. In the proof of Proposition 4.2, the dilation $u_{t}(x)=u(x / t)(t>0)$ plays an important role.

Remark 4.3. Both Propositions 4.1 and 4.2 are stated for odd nonlinearities $h(\xi)$. Since we just consider positive solutions, extending the nonlinearity $f(\xi)$ to an odd function on $\mathbf{R}$, we can apply Propositions 4.1 and 4.2 to our setting (See [JT1] for more details).

Now we give a proof of Lemma 3.4.

Proof of Lemma 3.4. We apply Proposition 4.1 with $H(\xi)=H\left(x_{0}, \xi\right)=-\frac{1}{2} V\left(x_{0}\right) \xi^{2}+$ $G\left(x_{0}, \xi\right)$. We can see that (h3) holds if and only if $x_{0} \in \Omega$. Thus (i) of Lemma 3.4 follows. Now assume that $v(y)$ is a non-zero critical point of $\Phi_{x_{0}}(v)$. Then we have $\Phi_{x_{0}}^{\prime}(v) v=0$, i.e.,

$$
\int_{\mathbf{R}^{N}}|\nabla v|^{2}+V\left(x_{0}\right) v^{2} d y-\int_{\mathbf{R}^{N}} g\left(x_{0}, v\right) v d y=0 .
$$

By Corollary 1.3 (i), we have

$$
\|v\|_{H^{1}\left(\mathbf{R}^{N}\right)}^{2}-\int_{\mathbf{R}^{N}} f(v) v d y \leq 0
$$

and, using (1.2), it follows that for any arbitrary $\delta>0$

$$
\begin{aligned}
\|v\|_{H^{1}\left(\mathbf{R}^{N}\right)}^{2} & \leq \delta\|v\|_{2}^{2}+C_{\delta}\|v\|_{s+1}^{s+1} \\
& \leq \frac{\delta}{V_{0}}\|v\|_{H^{1}\left(\mathbf{R}^{N}\right)}^{2}+C_{\delta} C_{s+1}^{\prime}\|v\|_{H^{1}\left(\mathbf{R}^{N}\right)}^{s+1}
\end{aligned}
$$

Thus $\frac{1}{2}\|v\|_{H^{1}\left(\mathbf{R}^{N}\right)} \leq C_{\delta} C_{s+1}^{\prime}\|v\|_{H^{1}\left(\mathbf{R}^{N}\right)}^{s+1}$ and there exists $\delta_{1}>0$ such that $\|v\|_{H^{1}\left(\mathbf{R}^{N}\right)} \geq \delta_{1}$ for any non-zero critical point $v$ and $x_{0} \in \mathbf{R}^{N}$. Since $|v|_{x_{0}} \geq\|v\|_{H^{1}\left(\mathbf{R}^{N}\right)}$, we get (ii). 
For $x \in \mathbf{R}^{N}$ we set

$$
m(x)= \begin{cases}\text { least energy level of } \Phi_{x}(v) & \text { if } x \in \Omega \\ \infty & \text { if } x \in \mathbf{R}^{N} \backslash \Omega\end{cases}
$$

By Proposition 4.2, $m(x)$ is equal to the mountain pass value for $\Phi_{x}(v)$ if $x \in \Omega$. We have the following

Proposition 4.4. $m\left(x_{0}\right)=\inf _{x \in \mathbf{R}^{N}} m(x)$ if and only if $x_{0} \in \Lambda$ and $V\left(x_{0}\right)=\inf _{x \in \Lambda} V(x)$. In particular, $m(0)=\inf _{x \in \mathbf{R}^{N}} m(x)$.

Proof. Suppose that $x_{0} \in \Lambda$ satisfies $V\left(x_{0}\right)=\inf _{x \in \Lambda} V(x)$. By our choice of $\Lambda^{\prime}$ and $\chi$, we have $x_{0} \in \Lambda^{\prime}$ and $\chi\left(x_{0}\right)=1$. We also have $x_{0} \in \Omega$ by Remark 3.1. Using $V(x) \geq V\left(x_{0}\right)$ in $\Lambda, G(x, \xi) \leq F(\xi)$ for all $(x, \xi)$, we have for any $x \in \Omega$,

$$
\begin{aligned}
\Phi_{x}(v) & =\frac{1}{2}\|\nabla v\|_{2}^{2}+\frac{1}{2} V(x)\|v\|_{2}^{2}-\int_{\mathbf{R}^{N}} G(x, v) d y \\
& \geq \frac{1}{2}\|\nabla v\|_{2}^{2}+\frac{1}{2} V\left(x_{0}\right)\|v\|_{2}^{2}-\int_{\mathbf{R}^{N}} F(v) d y \\
& =\Phi_{x_{0}}(v) \quad \text { for all } v \in H^{1}\left(\mathbf{R}^{N}\right) .
\end{aligned}
$$

(We remark that this inequality is strict if $V(x)>V\left(x_{0}\right)$ and $v \not \equiv 0$.) Thus $m\left(x_{0}\right) \leq m(x)$ for all $x \in \mathbf{R}^{N}$.

Next suppose that $x^{\prime} \in \Lambda$ satisfies $V\left(x^{\prime}\right)>V\left(x_{0}\right)$. We take a path $\gamma \in \Gamma$ such that (4.4)-(4.5) are satisfied for $\tilde{I}(v)=\Phi_{x^{\prime}}(v)$. Then

$$
m\left(x_{0}\right) \leq \max _{t \in[0,1]} \Phi_{x_{0}}(\gamma(t))<\max _{t \in[0,1]} \Phi_{x^{\prime}}(\gamma(t))=m\left(x^{\prime}\right)
$$

Therefore Proposition 4.4 holds.

We end this section establishing the continuity of $m(x)$.

Proposition 4.5. The function $m(x): \mathbf{R}^{N} \rightarrow(-\infty, \infty]$ is continuous in the following sense :

$$
\begin{aligned}
& m\left(x_{j}\right) \rightarrow m\left(x_{0}\right) \quad \text { if } x_{j} \rightarrow x_{0} \in \Omega, \\
& m\left(x_{j}\right) \rightarrow \infty \quad \text { if } x_{j} \rightarrow x_{0} \in \mathbf{R}^{N} \backslash \Omega .
\end{aligned}
$$

Proof. We make use of Propositions 4.1 and 4.2. First we deal with the case $x_{0} \in \Omega$ and suppose $\left(x_{j}\right) \subset \Omega$ satisfies $x_{j} \rightarrow x_{0} \in \Omega$. The upper semi-continuity

$$
\limsup _{j \rightarrow \infty} m\left(x_{j}\right) \leq m\left(x_{0}\right)
$$


is a consequence of the mountain pass characterization of $m(x)$. Indeed, for $x_{0} \in \Omega$ there exists a path $\gamma(t) \in C\left([0,1], H^{1}\left(\mathbf{R}^{N}\right)\right)$ such that

$$
\begin{aligned}
& \gamma(0)=0, \\
& \Phi_{x_{0}}(\gamma(1))<0, \\
& \max _{t \in[0,1]} \Phi_{x_{0}}(\gamma(t))=m\left(x_{0}\right) .
\end{aligned}
$$

Since $x_{j} \rightarrow x_{0},(4.7)$ and (4.8) hold for $\Phi_{x_{j}}(v)$ if $j$ is sufficiently large and

$$
m\left(x_{j}\right) \leq \max _{t \in[0,1]} \Phi_{x_{j}}(\gamma(t)) \rightarrow m\left(x_{0}\right) \quad \text { as } j \rightarrow \infty .
$$

To show the lower semi-continuity $\liminf _{j \rightarrow \infty} m\left(x_{j}\right) \geq m\left(x_{0}\right)$, it suffices to show that for least energy solutions $u_{j}(y)$ of $\Phi_{x_{j}}(v)$ one has

(i) $\left\|u_{j}\right\|_{H^{1}\left(\mathbf{R}^{N}\right)}$ is bounded as $j \rightarrow \infty$.

(ii) After extracting a subsequence, $u_{j}$ has a non-zero weak limit $u_{0}(y)$ and

$$
\liminf _{j \rightarrow \infty} \Phi_{x_{j}}\left(u_{j}\right) \geq \Phi_{x_{0}}\left(u_{0}\right) .
$$

Indeed it is then easily seen that the weak limit $u_{0}(y)$ is a non-zero critical point of $\Phi_{x_{0}}(v)$ and thus we have

$$
\liminf _{j \rightarrow \infty} m\left(x_{j}\right)=\liminf _{j \rightarrow \infty} \Phi_{x_{j}}\left(u_{j}\right) \geq \Phi_{x_{0}}\left(u_{0}\right) \geq m\left(x_{0}\right) .
$$

The proof of (i)-(ii) consists of several steps. First we remark that we may assume $u_{j}(y)$ to be radially symmetric with respect to 0 . We also recall that $u_{j}(x)$ satisfies the Pohozaev identity :

$$
\frac{N-2}{2}\left\|\nabla u_{j}\right\|_{2}^{2}=N \int_{\mathbf{R}^{N}} H\left(x_{j}, u_{j}(y)\right) d y .
$$

Step 1: There exist $m_{0}, m_{1}>0$ independent of $j$ such that

$$
m_{0} \leq m\left(x_{j}\right) \leq m_{1} \quad \text { for all } j \in \mathbf{N} .
$$

The existence of the uniform upper bound $m_{1}$ follows from (4.6). For $m_{0}$, we observe that

$$
\Phi_{x_{j}}(v) \geq \frac{1}{2}\|\nabla v\|_{2}^{2}+\frac{1}{2} V_{0}\|v\|_{2}^{2}-\int_{\mathbf{R}^{N}} F(v) d y .
$$


Thus choosing $m_{0}$ to be the mountain pass value of $v \mapsto \frac{1}{2}\|\nabla v\|_{2}^{2}+\frac{1}{2} V_{0}\|v\|_{2}^{2}-\int_{\mathbf{R}^{N}} F(v) d y$, we get the conclusion of Step 1.

Step 2: $\left\|\nabla u_{j}\right\|_{2}^{2} \in\left[N m_{0}, N m_{1}\right]$.

Using the Pohozaev identity (4.9), we have

$$
m\left(x_{j}\right)=\frac{1}{2}\left\|\nabla u_{j}\right\|_{2}^{2}-\int_{\mathbf{R}^{N}} H\left(x_{j}, u_{j}(y)\right) d y=\frac{1}{N}\left\|\nabla u_{j}\right\|_{2}^{2} .
$$

Thus the conclusion of Step 2 follows from Step 1.

Step 3 : Boundedness of $\left\|u_{j}\right\|_{2}$ for $N \geq 3$.

By Step 2, we can see that $\int_{\mathbf{R}^{N}} H\left(x_{j}, u_{j}\right) d y=-\frac{1}{2} V\left(x_{j}\right)\left\|u_{j}\right\|_{2}^{2}+\int_{\mathbf{R}^{N}} G\left(x_{j}, u_{j}\right) d y$ is bounded as $j \rightarrow \infty$. Thus there exists $C>0$ such that

$$
\begin{aligned}
\frac{1}{2} V\left(x_{j}\right)\left\|u_{j}\right\|_{2}^{2} & \leq C+\int_{\mathbf{R}^{N}} G\left(x_{j}, u_{j}\right) d y \\
& \leq C+\int_{\mathbf{R}^{N}} F\left(u_{j}\right) d y .
\end{aligned}
$$

By (f2), for any $\delta>0$ we can find $C_{\delta}>0$ such that

$$
|F(\xi)| \leq \delta|\xi|^{2}+C_{\delta}|\xi|^{\frac{2 N}{N-2}} .
$$

Thus

$$
\frac{1}{2} V\left(x_{j}\right)\left\|u_{j}\right\|_{2}^{2} \leq C+\delta\left\|u_{j}\right\|_{2}^{2}+C_{\delta}\left\|u_{j}\right\|_{\frac{2 N}{N-2}}^{\frac{2 N}{N-2}} .
$$

By the Sobolev embedding $\left\|u_{j}\right\|_{\frac{2 N}{N-2}} \leq C\left\|\nabla u_{j}\right\|_{2},\left\|u_{j}\right\|_{\frac{2 N}{N-2}}$ is bounded and choosing $\delta>0$ small, we get the boundedness of $\left\|u_{j}\right\|_{2}$.

Step 4: Boundedness of $\left\|u_{j}\right\|_{2}$ for $N=2$.

When $N=2$, the proof of the boundedness of $\left\|u_{j}\right\|_{2}$ is more technical. We argue indirectly and assume that $\left\|u_{j}\right\|_{2} \rightarrow \infty$. We set $t_{j}=\frac{1}{\left\|u_{j}\right\|_{2}} \rightarrow 0$ and $\tilde{u}_{j}(y)=u_{j}\left(y / t_{j}\right)$. Then we have

$$
\begin{aligned}
& \left\|\nabla \tilde{u}_{j}\right\|_{2}=\left\|\nabla u_{j}\right\|_{2} \in\left[2 m_{0}, 2 m_{1}\right], \\
& \left\|\tilde{u}_{j}\right\|_{2}=1 .
\end{aligned}
$$

We claim that $\tilde{u}_{j} \rightarrow 0$ weakly in $H^{1}\left(\mathbf{R}^{2}\right)$. In fact, suppose that $\tilde{u}_{j} \rightarrow \tilde{u}_{0}$ after extracting a subsequence. Since $u_{j}(y)$ is a critical point of $\Phi_{x_{j}}(v)$, we have

$$
-t_{j}^{2} \Delta \tilde{u}_{j}+V\left(x_{j}\right) \tilde{u}_{j}=g\left(x_{j}, \tilde{u}_{j}\right) \text { in } \mathbf{R}^{2} .
$$

Thus, passing to the limit as $j \rightarrow \infty$, we obtain

$$
V\left(x_{0}\right) \tilde{u}_{0}(y)=g\left(x_{0}, \tilde{u}_{0}(y)\right) \text { in } \mathbf{R}^{2} .
$$

Since $\tilde{u}_{0} \in H^{1}\left(\mathbf{R}^{2}\right)$ and $0 \in \mathbf{R}$ is an isolated solution of $V\left(x_{0}\right) \xi=g\left(x_{0}, \xi\right)$, this shows that $\tilde{u}_{0}(y) \equiv 0$.

Now we recall the following lemma. 
Lemma 4.6. ([BL]). Suppose $N \geq 2$. Then there exists a constant $C_{N}>0$ such that for any radially symmetric function $u(y) \in H^{1}\left(\mathbf{R}^{N}\right)$

$$
|u(x)| \leq C_{N}\|u\|_{H^{1}\left(\mathbf{R}^{N}\right)}|x|^{-\frac{N-1}{2}} \quad \text { for all }|x| \geq 1
$$

Applying Lemma 4.6 to $\tilde{u}_{j}(y)$, we get $\left|\tilde{u}_{j}(y)\right| \leq C^{\prime}|y|^{-\frac{1}{2}}$ for $|y| \geq 1$ and we can fix $R>1$ such that

$$
\left|\frac{g\left(x_{j}, \tilde{u}_{j}(y)\right)}{\tilde{u}_{j}(y)}\right| \leq \frac{1}{2} V_{0} \quad \text { for all } j \in \mathbf{N} \text { and }|y| \geq R
$$

Multiplying (4.12) by $\tilde{u}_{j}$ and integrating over $\mathbf{R}^{2}$, we get

$$
\begin{aligned}
t_{j}^{2}\left\|\nabla \tilde{u}_{j}\right\|_{2}^{2} & +\int_{|y| \geq R}\left(V\left(x_{j}\right)-\frac{g\left(x_{j}, \tilde{u}_{j}(y)\right)}{\tilde{u}_{j}(y)}\right) \tilde{u}_{j}^{2}(y) d y \\
& \leq-\int_{|y| \leq R} V\left(x_{j}\right) \tilde{u}_{j}^{2}(y)-g\left(x_{j}, \tilde{u}_{j}(y)\right) \tilde{u}_{j}(y) d y
\end{aligned}
$$

Since $\tilde{u}_{j} \rightarrow \tilde{u}_{0} \equiv 0$ weakly in $H^{1}\left(\mathbf{R}^{2}\right)$, and thus strongly in $L^{s+1}(|y| \leq R)$, we can see $\int_{|y| \geq R}\left(V\left(x_{j}\right)-\frac{g\left(x_{j}, \tilde{u}_{j}(y)\right)}{\tilde{u}_{j}(y)}\right) \tilde{u}_{j}^{2}(y) d y \rightarrow 0$. Using (4.13), we get $\left\|\tilde{u}_{j}\right\|_{L^{2}(|y| \geq R)} \rightarrow 0$, that is, $\left\|\tilde{u}_{j}\right\|_{2} \rightarrow 0$. But this contradicts (4.11). Thus the proof of Step 4 is completed.

Step 5: After extracting a subsequence, $u_{j}$ has a non-zero weak limit $u_{0}(y) \not \equiv 0$.

We remark that the boundedness of $\left\|u_{j}\right\|_{H^{1}\left(\mathbf{R}^{N}\right)}$ follows from Steps 2-4. Here we argue indirectly as in Step 4. We assume that $u_{j} \rightarrow 0$ weakly in $H^{1}\left(\mathbf{R}^{N}\right)$ and strongly in $L_{l o c}^{s+1}\left(\mathbf{R}^{N}\right)$. Using Lemma 4.6 , we can find a $R>1$ such that

$$
\left|\frac{g\left(x_{j}, u_{j}(y)\right)}{u_{j}(y)}\right| \leq \frac{1}{2} V_{0} \quad \text { for all } j \in \mathbf{N} \text { and }|y| \geq R .
$$

It follows from $\Phi_{x_{j}}^{\prime}\left(u_{j}\right) u_{j}=0$ that

$$
\begin{aligned}
\left\|\nabla u_{j}\right\|_{2}^{2} & +\int_{|y| \geq R}\left(V\left(x_{j}\right)-\frac{g\left(x_{j}, u_{j}(y)\right)}{u_{j}(y)}\right) u_{j}^{2}(y) d y \\
& \leq-\int_{|y| \leq R} V\left(x_{j}\right) u_{j}^{2}(y)-g\left(x_{j}, u_{j}(y)\right) u_{j}(y) d y
\end{aligned}
$$

That is,

$$
\left\|\nabla u_{j}\right\|_{2}^{2} \leq-\int_{|y| \leq R} V\left(x_{j}\right) u_{j}^{2}(y)-g\left(x_{j}, u_{j}(y)\right) u_{j}(y) d y
$$

and $u_{j} \rightarrow 0$ in $L_{l o c}^{s+1}\left(\mathbf{R}^{N}\right)$ implies $\left\|\nabla u_{j}\right\|_{2} \rightarrow 0$. This is in contradiction with Step 2 . 
Step $6: \liminf _{j \rightarrow \infty} \Phi_{x_{j}}\left(u_{j}\right) \geq \Phi_{x_{0}}\left(u_{0}\right)$.

Since we are working in a space of radially symmetric functions, we can see from (f1)-(f2) that

$$
\int_{\mathbf{R}^{N}} G\left(x_{j}, u_{j}\right) d y \rightarrow \int_{\mathbf{R}^{N}} G\left(x_{0}, u_{0}\right) d y \quad \text { as } j \rightarrow \infty .
$$

(See Theorem A.I of $[\mathbf{B L}]$ ). Thus we have the desired result from the lower semi-continuity of the $L^{2}$-norm.

Finally we deal with the case $x_{0} \notin \Omega$.

Step $7:$ Suppose $x_{0} \notin \Omega$ and $x_{j} \rightarrow x_{0}$. Then $m\left(x_{j}\right) \rightarrow \infty$.

In fact, if $m\left(x_{j}\right) \nrightarrow \infty$, we can find a subsequence - still denoted by $x_{j}$ - such that $m\left(x_{j}\right)$ stays bounded as $j \rightarrow \infty$. Then by the arguments of Steps $1-5$, we can find a non-zero critical point of $\Phi_{x_{0}}(v)$. However it is a contradiction to Lemma 3.4 (i). 


\section{End of the proof of Theorem 0.1}

In this section we conclude the proof of Theorem 0.1. First we study the behavior of $b_{\varepsilon}$ as $\varepsilon \rightarrow 0$.

Proposition 5.1. Let $\left(b_{\varepsilon}\right)_{\varepsilon \in\left(0, \varepsilon_{1}\right]}$ be the mountain pass value of $J_{\varepsilon}(v)$ defined in (1.21)(1.22). Then

$$
b_{\varepsilon} \rightarrow m(0)=\inf _{x \in \mathbf{R}^{N}} m(x) \quad \text { as } \varepsilon \rightarrow 0 .
$$

Proof. By Proposition 4.2 there exists a path $\gamma \in C\left([0,1], H^{1}\left(\mathbf{R}^{N}\right)\right)$ such that

$$
\begin{aligned}
& \gamma(0)=0, \Phi_{0}(\gamma(1))<0, \\
& \Phi_{0}(\gamma(t)) \leq m(0) \text { for all } t \in[0,1], \\
& \max _{t \in[0,1]} \Phi_{0}(\gamma(t))=m(0) .
\end{aligned}
$$

Let $\varphi(y) \in C_{0}^{\infty}\left(\mathbf{R}^{N}\right)$ be such that $\varphi(0)=1$ and $\varphi \geq 0$. Setting

$$
\gamma_{R}(t)(y)=\varphi(y / R) \gamma(t)(y)
$$

we have $\gamma_{R}(t) \in C\left([0,1], H_{\varepsilon}\right), \gamma_{R}(0)=0$ and $\Phi_{0}\left(\gamma_{R}(1)\right)<0$ for sufficiently large $R>1$. Then, in particular, $\gamma_{R}(t) \in \Gamma_{\varepsilon}$. Also for any fixed $R>0$,

$$
J_{\varepsilon}\left(\gamma_{R}(t)\right) \rightarrow \Phi_{0}\left(\gamma_{R}(t)\right) \quad \text { as } \varepsilon \rightarrow 0 \text { uniformly in } t \in[0,1] .
$$

Thus for sufficiently large $R>1$

$$
b_{\varepsilon} \leq \max _{t \in[0,1]} J_{\varepsilon}\left(\gamma_{R}(t)\right) \rightarrow \max _{t \in[0,1]} \Phi_{0}\left(\gamma_{R}(t)\right) \quad \text { as } \varepsilon \rightarrow 0 .
$$

Since

$$
\max _{t \in[0,1]} \Phi_{0}\left(\gamma_{R}(t)\right) \rightarrow m(0) \quad \text { as } R \rightarrow \infty,
$$

we deduce from (5.2) that

$$
\limsup _{\varepsilon \rightarrow 0} b_{\varepsilon} \leq m(0) .
$$

Now let $v_{\varepsilon} \in H_{\varepsilon}$ be a critical point of $J_{\varepsilon}(v)$ associated to $b_{\varepsilon}$. Applying Proposition 3.2, we can find $\varepsilon_{j} \rightarrow 0, \ell \in \mathbf{N} \cup\{0\},\left(y_{\varepsilon_{j}}^{k}\right), x^{k}, \omega^{k}(k=1,2, \cdots, \ell)$ satisfying (CC.1)-(CC.5). If we assume that $\ell=0$, then (CC.5) implies that $b_{\varepsilon_{j}}=J_{\varepsilon_{j}}\left(v_{\varepsilon_{j}}\right) \rightarrow 0$ in contradiction with (1.23). Thus $\ell \geq 1$ and again from (CC.5) it follows that

$$
\lim _{j \rightarrow \infty} b_{\varepsilon_{j}}=\sum_{k=1}^{\ell} \Phi_{x^{k}}\left(\omega^{k}\right) \geq \sum_{k=1}^{\ell} m\left(x^{k}\right) \geq \ell m(0) \geq m(0) .
$$


Thus combining (5.3) and (5.4), we get (5.1).

As a consequence of Proposition 5.1 we have :

Proposition 5.2. For any $\varepsilon \in\left(0, \varepsilon_{1}\right]$ let $\left(v_{\varepsilon}\right)$ denote a critical point of $J_{\varepsilon}(v)$ corresponding to $b_{\varepsilon}$. Then for any sequence $\varepsilon_{j} \rightarrow 0$ there exist a subsequence — still denoted by $\varepsilon_{j}$ and $\left(y_{\varepsilon_{j}}\right), x^{1}, \omega^{1}$ such that

(i) $\varepsilon_{j} y_{\varepsilon_{j}} \rightarrow x^{1}$.

(ii) $x^{1} \in \Lambda^{\prime}$ satisfies $V\left(x^{1}\right)=\inf _{x \in \Lambda} V(x)$.

(iii) $\omega^{1}(y)$ is a least energy solution of $\Phi_{x^{1}}^{\prime}(v)=0$.

(iv) $\left\|v_{\varepsilon_{j}}-\psi_{\varepsilon_{j}} \omega^{1}\left(y-y_{\varepsilon_{j}}\right)\right\|_{H_{\varepsilon_{j}}} \rightarrow 0$.

(v) $J_{\varepsilon_{j}}\left(v_{\varepsilon_{j}}\right) \rightarrow m\left(x^{1}\right)=m(0)$.

Proof. Arguing as in the proof of Proposition 5.1 it follows that $\ell=1$ in Proposition 3.2. Hence we have (5.5)-(5.9).

Now we are ready to give the proof of Theorem 0.1 .

Proof of Theorem 0.1. We divide the proof of Theorem 0.1 into several steps. In what follows, $v_{\varepsilon}(y) \in H_{\varepsilon}$ denotes a critical point corresponding to $b_{\varepsilon}$. We shall prove that this is a desired solution when $\varepsilon>0$ is small enough. For this it suffices to show that for any sequence $\varepsilon_{j} \rightarrow 0$ there exists a subsequence — still denoted by $\varepsilon_{j}$ — such that for large $j, v_{\varepsilon_{j}}$ takes a unique local maximum at $\bar{x}_{\varepsilon_{j}} \in \Lambda / \varepsilon_{j}$ with $V\left(\varepsilon_{j} \bar{x}_{\varepsilon_{j}}\right) \rightarrow \inf _{x \in \Lambda} V(x)$ and decreases sufficiently fast away from $\bar{x}_{\varepsilon_{j}}$.

Let $\varepsilon_{j} \rightarrow 0$ be an arbitrary fixed sequence. Applying Proposition 5.2 we can assume that there exists $\left(y_{\varepsilon_{j}}\right), x^{1}, \omega^{1}$ such that (5.5)-(5.9) hold. Moreover, by the maximum principle, $v_{\varepsilon}(y) \geq 0$ for all $y \in \mathbf{R}^{N}$.

Step 1 : If a sequence $\left(z_{\varepsilon_{j}}\right) \subset \mathbf{R}^{N}$ satisfies

$$
\liminf _{j \rightarrow \infty} \int_{B_{1}\left(z_{\varepsilon_{j}}\right)}\left|v_{\varepsilon_{j}}\right|^{2} d y>0
$$

then $\lim \sup _{j \rightarrow \infty}\left|z_{\varepsilon_{j}}-y_{\varepsilon_{j}}\right|<\infty$. In particular we have $\lim _{j \rightarrow \infty}\left|\varepsilon_{j} z_{\varepsilon_{j}}-x^{1}\right|=0$. Conversely if $\left(z_{\varepsilon_{j}}\right)$ satisfies $\left|z_{\varepsilon_{j}}-y_{\varepsilon_{j}}\right| \rightarrow \infty$, we have $\int_{B_{1}\left(z_{\varepsilon_{j}}\right)}\left|v_{\varepsilon_{j}}\right|^{2} d y \rightarrow 0$.

This clearly follows from $(5.5)$, (5.8).

Step $2: \sup _{z \in\left(\bar{\Lambda} \backslash \Lambda^{\prime}\right) / \varepsilon_{j}}\left|v_{\varepsilon_{j}}(z)\right| \rightarrow 0$ as $j \rightarrow \infty$.

It follows from Step 1 that

$$
\sup _{z \in\left(\bar{\Lambda} \backslash \Lambda^{\prime}\right) / \varepsilon_{j}} \int_{B_{1}(z)}\left|v_{\varepsilon_{j}}\right|^{2} d y \rightarrow 0 \quad \text { as } j \rightarrow \infty .
$$


It also follows from the boundedness of $\left(v_{\varepsilon_{j}}\right)$ in $H^{1}\left(\mathbf{R}^{N}\right)$ that

$$
\left\|v_{\varepsilon_{j}}\right\|_{L^{s+1}\left(B_{1}(z)\right)} \rightarrow 0 \quad \text { uniformly in } z \in\left(\bar{\Lambda} \backslash \Lambda^{\prime}\right) / \varepsilon_{j}
$$

We remark that $V\left(\varepsilon_{j} y\right), \chi\left(\varepsilon_{j} y\right)$ stay bounded uniformly in $\left(\bar{\Lambda} \backslash \Lambda^{\prime}\right) / \varepsilon_{j}$ as $j \rightarrow \infty$. Thus since $v_{\varepsilon_{j}}(y)$ is a solution of

$$
-\Delta v+V\left(\varepsilon_{j} y\right) v=g\left(\varepsilon_{j} y, v\right) \quad \text { in } B_{1}(z) .
$$

By standard regularity arguments we have $v_{\varepsilon_{j}}(y) \in C\left(B_{1}(z)\right)$, and (5.11) implies

$$
\left\|v_{\varepsilon_{j}}\right\|_{L^{\infty}\left(B_{1}(z)\right)} \rightarrow 0 \quad \text { as } j \rightarrow \infty
$$

uniformly in $z \in\left(\bar{\Lambda} \backslash \Lambda^{\prime}\right) / \varepsilon_{j}$.

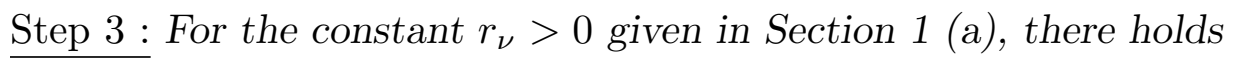

$$
v_{\varepsilon_{j}}(y) \leq r_{\nu} \quad \text { in } \mathbf{R}^{N} \backslash\left(\Lambda^{\prime} / \varepsilon_{j}\right)
$$

By Step 2, $\sup _{z \in\left(\bar{\Lambda} \backslash \Lambda^{\prime}\right) / \varepsilon_{j}}\left|v_{\varepsilon_{j}}(y)\right| \leq \frac{r_{\nu}}{2}$ for small $\varepsilon_{j}$. Since $\left.\left(v_{\varepsilon_{j}}(y)-r_{\nu}\right)_{+}\right|_{\mathbf{R}^{N} \backslash\left(\Lambda^{\prime} / \varepsilon_{j}\right)} \in H_{\varepsilon}$ it follows from $J_{\varepsilon}^{\prime}\left(v_{\varepsilon_{j}}\right)\left(\left.\left(v_{\varepsilon_{j}}(y)-r_{\nu}\right)_{+}\right|_{\mathbf{R}^{N} \backslash\left(\Lambda^{\prime} / \varepsilon_{j}\right)}\right)=0$ that

$$
\int_{\mathbf{R}^{N} \backslash\left(\Lambda^{\prime} / \varepsilon_{j}\right)}\left|\nabla\left(v_{\varepsilon_{j}}-r_{\nu}\right)_{+}\right|^{2}+V\left(\varepsilon_{j} y\right) v_{\varepsilon_{j}}\left(v_{\varepsilon_{j}}-r_{\nu}\right)_{+}-\underline{f}\left(v_{\varepsilon_{j}}\right)\left(v_{\varepsilon_{j}}-r_{\nu}\right)_{+} d y=0 .
$$

By Lemma 1.2 (ii),

$$
\int_{\mathbf{R}^{N} \backslash\left(\Lambda^{\prime} / \varepsilon_{j}\right)}\left|\nabla\left(v_{\varepsilon_{j}}-r_{\nu}\right)_{+}\right|^{2}+\left(V_{0}-\nu\right) v_{\varepsilon_{j}}\left(v_{\varepsilon_{j}}-r_{\nu}\right)_{+} d y \leq 0
$$

Thus $\left(v_{\varepsilon_{j}}-r_{\nu}\right)_{+} \equiv 0$ in $\mathbf{R}^{N} \backslash\left(\Lambda^{\prime} / \varepsilon_{j}\right)$. That is, (5.12) holds.

By Step 3 we see that $v_{\varepsilon_{j}}(y)$ is a solution of the rescaled original problem :

$$
-\Delta v+V\left(\varepsilon_{j} y\right) v=f(v) \text { in } \mathbf{R}^{N}
$$

for sufficiently small $\varepsilon_{j}>0$. Since $f(\xi) \in C^{1}\left(\mathbf{R}^{N}, \mathbf{R}\right)$, we have $v_{\varepsilon_{j}}(y) \in C^{2}\left(\mathbf{R}^{N}\right)$ from a standard regularity argument. From the boundedness of $\left\|v_{\varepsilon_{j}}\right\|_{H_{\varepsilon}}$ we can see also that $\left\|v_{\varepsilon_{j}}\right\|_{C^{2}\left(K / \varepsilon_{j}\right)}$ is bounded on any compact set $K \subset \mathbf{R}^{N}$ as $j \rightarrow \infty$. We remark that $V\left(\varepsilon_{j} y\right)$ and $\chi\left(\varepsilon_{j} y\right)$ stay bounded uniformly in $K / \varepsilon_{j}$ as $j \rightarrow \infty$. 
Step 4 : Suppose that $v_{\varepsilon_{j}}(y)$ takes a local maximum at $z_{\varepsilon_{j}}$. Then $\left(z_{\varepsilon_{j}}\right)$ satisfies

$$
\limsup _{j \rightarrow \infty}\left|z_{\varepsilon_{j}}-y_{\varepsilon_{j}}\right|<\infty \quad \text { and } \quad \varepsilon_{j} z_{\varepsilon_{j}} \rightarrow x^{1}
$$

By the maximum principle, we see that $v_{\varepsilon_{j}}\left(z_{\varepsilon_{j}}\right) \geq r_{\nu}$. Since $v_{\varepsilon_{j}}(y)$ is bounded in $C_{l o c}^{2}$, we can also get $\liminf \operatorname{in}_{j \rightarrow \infty} \int_{B_{1}\left(z_{\varepsilon_{j}}\right)}\left|v_{\varepsilon_{j}}\right|^{2} d y>0$. We conclude by Step 1.

Step $5: v_{\varepsilon_{j}}$ has only one local maximum for $\varepsilon_{j}$ small.

Assume that $v_{\varepsilon_{j}}(y)$ takes a local maximum at $y=z_{\varepsilon_{j}}$. By the maximum principle, $v_{\varepsilon_{j}}\left(z_{\varepsilon_{j}}\right) \geq r_{\nu}$. Since $v_{\varepsilon_{j}}$ is bounded in $H^{1}\left(\mathbf{R}^{N}\right)$ and $C_{l o c}^{2}\left(\mathbf{R}^{N}\right)$, after extracting a subsequence, we may assume $v_{\varepsilon_{j}}\left(y+z_{\varepsilon_{j}}\right) \rightarrow \omega(y)$ weakly in $H^{1}\left(\mathbf{R}^{N}\right)$ and strongly in $C_{l o c}^{2}$ with $\omega(y)$ satisfying

$$
-\Delta \omega+V\left(x^{1}\right) \omega=f(\omega) \text { in } \mathbf{R}^{N}
$$

and having a local maximum at $y=0$. Thus by the result of $[\mathbf{G N N}], \omega(y)$ is radially symmetric with respect to 0 and strictly decreasing with respect to $r=|y|$. Thus if $v_{\varepsilon_{j}}(y)$ takes two local maxima at $y=z_{\varepsilon_{j}}$ and $y=z_{\varepsilon_{j}}^{\prime}$, then we necessarily have $\left|z_{\varepsilon_{j}}-z_{\varepsilon_{j}}^{\prime}\right| \rightarrow \infty$. However Step 4 implies $\lim \sup \left|z_{\varepsilon_{j}}-z_{\varepsilon_{j}}^{\prime}\right| \leq \lim \sup \left|z_{\varepsilon_{j}}-y_{\varepsilon_{j}}\right|+\lim \sup \left|z_{\varepsilon_{j}}^{\prime}-y_{\varepsilon_{j}}\right|<\infty$. This contradiction shows that $v_{\varepsilon_{j}}(y)$ takes only one local maximum.

Step 6 : There exists $\ell_{0}>0$ such that for small $\varepsilon_{j}>0$

$$
\left|v_{\varepsilon_{j}}(y)\right|<r_{\nu} \quad \text { for all }\left|y-\bar{x}_{\varepsilon_{j}}\right| \geq \ell_{0}
$$

where $\bar{x}_{\varepsilon_{j}}$ is the unique local maximum of $v_{\varepsilon_{j}}(y)$.

Indeed, if $z_{\varepsilon_{j}}$ satisfies $v_{\varepsilon_{j}}\left(z_{\varepsilon_{j}}\right) \geq r_{\nu}$, then we have $\liminf \inf _{j \rightarrow \infty} \int_{B_{1}\left(z_{\varepsilon_{j}}\right)}\left|v_{\varepsilon_{j}}\right|^{2} d y>0$ and Steps 1,4 implies that $\lim \sup \left|z_{\varepsilon_{j}}-\bar{x}_{\varepsilon_{j}}\right| \leq \lim \sup \left|z_{\varepsilon_{j}}-y_{\varepsilon_{j}}\right|+\lim \sup \left|y_{\varepsilon_{j}}-\bar{x}_{\varepsilon_{j}}\right|<\infty$. Thus there is no sequence $\left(z_{\varepsilon_{j}}\right)$ satisfying $\left|z_{\varepsilon_{j}}-\bar{x}_{\varepsilon_{j}}\right| \rightarrow \infty$ and $v_{\varepsilon_{j}}\left(z_{\varepsilon_{j}}\right) \geq r_{\nu}$. Step 6 follows.

Step 7 : Conclusion.

Consider the unique solution $\eta(y) \in H^{1}\left(|y| \geq \ell_{0}\right)$ of the following problem :

$$
\begin{array}{cc}
-\Delta \eta+\frac{V_{0}}{2} \eta=0 & \text { in }|y| \geq \ell_{0}, \\
\eta(y)=r_{\nu} & \text { on }|y|=\ell_{0} .
\end{array}
$$

It is easily seen that $\eta(y)$ has an exponential decay and since $\frac{f\left(v_{\varepsilon_{j}}(y)\right)}{v_{\varepsilon_{j}}(y)} \leq \frac{V_{0}}{2}$ when $|y| \geq \ell_{0}$, we have, by the maximum principle that $v_{\varepsilon_{j}}\left(y+\bar{x}_{\varepsilon_{j}}\right) \leq \eta(y)$ for $|y| \geq \ell_{0}$. Thus $v_{\varepsilon_{j}}(y)$ also has an exponential decay. 
Now setting $u_{\varepsilon_{j}}(x)=v_{\varepsilon_{j}}\left(x / \varepsilon_{j}\right)$ we can easily see that $u_{\varepsilon_{j}}(x)$ has the desired properties. This concludes the proof of Theorem 0.1.

Acknowledgements. A part of this paper was written during the second Author was visiting the Laboratoire de Mathématiques of the University of Franche-Comté. He would like to thank the University of Franche-Comté for support and hospitality. The second author was also partially supported by the Japan Association of Promotion of Science and Waseda University Grant for Special Research Projects. 


\section{References}

[ABC] A. Ambrosetti, M. Badiale and S. Cingolani, Semiclassical states of nonlinear Schrödinger equations. Arch. Rational Mech. Anal. 140 (1997), 285-300.

[BL] H. Berestycki and P. L. Lions, Nonlinear scalar field equations I, Arch. Rat. Mech. Anal. 82 (1983), 313-346.

[BGK] H. Berestycki, T. Gallouët and O. Kavian, Equations de Champs scalaires euclidiens non linéaires dans le plan. C. R. Acad. Sci; Paris Ser. I Math. 297 (1983), 307-310 and Publications du Laboratoire d'Analyse Numérique, Université de Paris VI (1984).

[Br] H. Brezis, Analyse fonctionnelle, Masson, (1983).

[DF1] M. del Pino and P. Felmer, Local mountain passes for semilinear elliptic problems in unbounded domains, Calc. Var. PDE 4 (1996), 121-137.

[DF2] M. del Pino and P. Felmer, Multi-peak bound states of nonlinear Schrödinger equations, Ann. IHP, Analyse Nonlineaire, 15 (1998), 127-149.

[DF3] M. del Pino and P. Felmer, Semi-classical states of nonlinear Schrödinger equations : a variational reduction method, Math. Ann. 324 (2002), no. 1, 1-32.

[DFT $]$ M. del Pino, P. Felmer and K. Tanaka, An elementary construction of complex patterns in nonlinear Schrödinger equations, Nonlinearity 15 (2002), no. 5, 1653-1671.

[FW] A. Floer and A. Weinstein, Nonspreading wave packets for the cubic Schrödinger equation with a bounded potential, J. Funct. Anal. 69 (1986), no. 3, 397-408.

[GNN] B. Gidas, W.-M. Ni and L. Nirenberg, Symmetry of positive solutions of nonlinear equations in $\mathbf{R}^{N}$, Math. Anal. and Applications, Part A, Advances in Math. Suppl. Studies 7A (ed. L. Nachbin), Academic Press, 369-402 (1981).

[Gr] M. Grossi, Some results on a class of nonlinear Schrödinger equations. Math. Zeit. 235 (2000), 687-705.

[Gu] C. Gui, Existence of multi-bump solutions for nonlinear Schrödinger equations via variational method. Comm. Partial Differential Equations 21 (1996), 787-820.

$[\mathbf{J}]$ L. Jeanjean, On the existence of bounded Palais-Smale sequences and applications to a Landesman-Lazer-type problem set on $\mathbf{R}^{N}$, Proc. Roy. Soc. Edinburgh 129A (1999), 787-809.

[JT1] L. Jeanjean and K. Tanaka, A positive solution for an asymptotically linear elliptic problem on $\mathbf{R}^{N}$ autonomous at infinity, ESAIM Control Optim. Calc. Var. 7 (2002), $597-614$.

[JT2] L. Jeanjean and K. Tanaka, A remark on least energy solutions in $\mathbf{R}^{N}$, Proc. Amer. Math. Soc. 131 (2003), 2399-2408. 
[KW] X. Kang and J. Wei, On interacting bumps of semi-classical states of nonlinear Schrödinger equations, Advances Diff. Eq. 5 (2000), 899-928.

[YYL] YanYan Li, On a singularly perturbed elliptic equation. Adv. Differential Equations 2 (1997), 955-980.

[L] P.-L. Lions, The concentration-compactness principle in the calculus of variations. The locally compact case. I. Ann. Inst. H. Poincaré Anal. Non Linéaire 1 (1984), 109-145 and II. Ann. Inst. H. Poincaré Anal. Non Linéaire 1 (1984), 223-283.

[NaT] K. Nakashima and K. Tanaka, Clustering layers and boundary layers in spatially inhomogeneous phase transition problems, Ann. I. H. Poincaré Anal. Non Linéaire 20 (2003), 107-143.

[NT1] W.-M. Ni and I. Takagi, On the shape of least-energy solutions to a semilinear Neumann problem, Comm. Pure Appl. Math. 44 (1991), 819-851.

[NT2] W.-M. Ni and I. Takagi, Locating the peaks of least-energy solutions to a semilinear Neumann problem, Duke Math. J. 70 (1993), 247-281.

[NW] W.-M. Ni and J. Wei, On the location and profile of spike-layer solutions to singularly perturbed semilinear Dirichlet problems, Comm. Pure Appl. Math. 48 (1995), 731765.

[O1] Y.-G. Oh, Existence of semiclassical bound states of nonlinear Schrödinger equations with potentials of the class $(V)_{a}$. Comm. Partial Differential Equations 13 (1988), no. 12, 1499-1519.

[O2] Y.-G. Oh, On positive multi-lump bound states of nonlinear Schrödinger equations under multiple well potential. Comm. Math. Phys. 131 (1990), no. 2, 223-253.

[P] A. Pistoia, Multi-peak solutions for a class of nonlinear Schrödinger equations, NoDEA Nonlinear Diff. Eq. Appl. 9 (2002), 69-91.

[R] P. Rabinowitz, On a class of nonlinear Schrödinger equations, Z. Angew Math Phys 43, (1992), 270-291.

[Str] M. Struwe, A global compactness result for elliptic boundary value problems involving limiting nonlinearities. Math. Z. 187 (1984), 511-517.

[Stu] C.A. Stuart, Personal communication, Summer 2000.

[W] X. Wang, On concentration of positive bound states of nonlinear Schrödinger equations, Comm. Math. Phys. 153 (1993), 229-244. 